\title{
Dispersion function for plasmas with loss-cone distributions in an inhomogeneous magnetic field
}

\author{
R. Gaelzer, R. S. Schneider, and L. F. Ziebell \\ Instituto de Física, UFRGS, Caixa Postal 15051, 91501-970 Porto Alegre, RS, Brazil
}

(Received 25 April 1996; revised manuscript received 13 November 1996)

\begin{abstract}
The dispersion relation for electromagnetic waves in a magnetized plasma with weakly inhomogeneous magnetic field is investigated within the framework of a WKB approximation. A dispersion function useful for the case of plasma particles described by a generalized loss-cone distribution is introduced, valid for waves propagating in weakly relativistic plasmas, for any direction relative to the ambient magnetic field and to the inhomogeneity. This dispersion function is in some particular cases related to other plasma dispersion functions well known from the study of homogeneous plasmas. An application is made for the case of ordinary mode waves propagating perpendicularly to the magnetic field in inhomogeneous loss-cone plasmas. [S1063-651X(97)07104-3]

PACS number(s): 52.25.Mq, 52.40.Db, 52.35.-g
\end{abstract}

\section{INTRODUCTION}

The study of wave propagation and absorption in inhomogeneous plasmas can be made by using a formulation in which the dielectric properties of the plasma are described by an effective dielectric tensor which incorporates inhomogeneity effects, inserted into a dispersion relation which is formally the same as that of a homogeneous plasma [1]. The fundamental features of the formalism were developed in a publication by Beskin, Gurevich, and Istomin (denoted in what follows as BGI), based upon the application of an iterative procedure to the wave equation, along with the requirement of energy conservation at all orders of the iteration [1]. The formulation is appropriate as long as the conditions of validity of the WKB approximation are satisfied.

We have recently utilized the BGI formalism in the study of electron cyclotron absorption in inhomogeneous media, both in the case of homogeneous magnetic field [2-4] and in the case of inhomogeneous magnetic field [5]. There is a fundamental difference between these two cases. The unperturbed orbits of the plasma particles are affected by the magnetic field inhomogeneity, while they are not affected by inhomogeneities in other plasma parameters such as density and temperature. The resonance condition in momentum space is therefore affected by the inhomogeneity, and according to the BGI procedure an infinite number of corrections is necessary to be added in order to build up the effective dielectric tensor, starting from a plane wave approximation [1].

In the present paper we introduce further developments in the derivation of the dielectric tensor for inhomogeneous magnetic field, and define a generalized dispersion function useful for the case of plasma particles described by a nonrelativistic loss-cone distribution of Dory-Guest-Harris (DGH) type [6]. We also discuss some of the properties of this dispersion function, particularly its relationship with other dispersion functions which appear in the treatment of homogeneous plasmas.

The plan of the paper is the following. In Sec. II we briefly describe the physical system to be considered and write the components of the effective dielectric tensor for electromagnetic waves in an inhomogeneous magnetized plasma, whose derivation is detailed elsewhere [5]. In Sec. III we consider the case of particles described by nonrelativistic DGH distributions, and introduce the generalized plasma dispersion function for inhomogeneous plasmas. Some properties of the inhomogeneous plasma dispersion function and several limit cases are discussed in Sec. IV, including its relationship with other well-known plasma dispersion functions appearing in the case of homogeneous plasmas. In Sec. V we illustrate the use of the generalized dispersion function by considering the case of ordinary mode waves propagating perpendicularly to the magnetic field in weakly inhomogeneous media. Finally, Sec. VI is reserved for the conclusions. Appendixes on the evaluation of relevant momentum integrals, on the resonance condition for particular cases, and on some characteristics of the anti-Hermitian part of the components of the effective dielectric tensor can be found at the end of the paper.

\section{THE DIELECTRIC TENSOR FOR INHOMOGENEOUS PLASMAS}

In a previous publication regarding the dielectric properties of inhomogeneous plasmas, we have utilized the BGI procedure and obtained explicit expressions for the components of the dielectric tensor for inhomogeneous plasmas which were written as

$$
\begin{aligned}
\overleftrightarrow{\varepsilon}= & \stackrel{1}{ }-i \sum_{\alpha} \frac{4 \pi q_{\alpha}^{2}}{m_{\alpha} \omega_{n \rightarrow-\infty}} \sum_{0}^{\infty} d \tau \\
& \times \int d^{3} u u_{\perp} \mathcal{L}\left(f_{\alpha 0}\right) e^{i D_{n \alpha} \tau} e^{-i n \psi_{n \alpha} \boldsymbol{\Pi}_{n \alpha}^{-} \boldsymbol{\Pi}_{n \alpha}^{+}} \\
& -\hat{\boldsymbol{e}}_{z} \hat{\boldsymbol{e}}_{z} \sum_{\alpha} \frac{4 \pi q_{\alpha}^{2}}{m_{\alpha} \omega^{2}} \int d^{3} u \frac{u_{\|}}{\gamma} L\left(f_{\alpha 0}\right),
\end{aligned}
$$




$$
\begin{aligned}
& \boldsymbol{\Pi}_{n \alpha}^{ \pm}=\frac{1}{\xi_{n}^{ \pm}}\left[\frac{n}{b_{\alpha} \xi_{n}^{ \pm}} J_{n}\left(b_{\alpha} \xi_{n}^{ \pm}\right)\left(\cos \psi \pm \frac{\epsilon_{n}}{k_{\perp}}\right)\right. \\
& \left.\mp i J_{n}^{\prime}\left(b_{\alpha} \xi_{n}^{ \pm}\right) \sin \psi\right] \hat{\boldsymbol{e}}_{x}+\frac{1}{\xi_{n}^{ \pm}}\left[\frac{n}{b_{\alpha} \xi_{n}^{ \pm}} J_{n}\left(b_{\alpha} \xi_{n}^{ \pm}\right) \sin \psi\right. \\
& \left. \pm i J_{n}^{\prime}\left(b_{\alpha} \xi_{n}^{ \pm}\right)\left(\cos \psi \pm \frac{\epsilon_{n}}{k_{\perp}}\right)\right] \hat{\boldsymbol{e}}_{y}+\frac{u_{\|}}{u_{\perp}} J_{n}\left(b_{\alpha} \xi_{n}^{ \pm}\right) \hat{\boldsymbol{e}}_{z}, \\
& D_{n \alpha}=\gamma \omega-k_{\|} c u_{\|}-n \Omega_{\alpha}(1+\epsilon x)-\epsilon \frac{k_{\perp} u_{\perp}^{2} c^{2}}{2 \Omega_{\alpha}} \sin \psi, \\
& \psi_{n \alpha}=\tan ^{-1}\left[\frac{2 \epsilon_{n} \sin \psi / k_{\perp}}{1-\left(\epsilon_{n} / k_{\perp}\right)^{2}}\right], \\
& \xi_{n}^{ \pm}=\left[\left(\cos \psi \pm \frac{\epsilon_{n}}{k_{\perp}}\right)^{2}+\sin ^{2} \psi\right]^{1 / 2}, \\
& b_{\alpha}=\frac{k_{\perp} u_{\perp} c}{\Omega_{\alpha}}, \\
& \epsilon_{n}=\epsilon n \Omega_{\alpha} \tau / 2, \\
& \mathcal{L}\left(f_{\alpha 0}\right)=\partial_{u_{\perp}} f_{0}-\frac{N_{\|} u_{\perp}}{\gamma} L\left(f_{\alpha 0}\right), \\
& L\left(f_{\alpha 0}\right)=\frac{u_{\|}}{u_{\perp}} \partial_{u_{\perp}} f_{0}-\partial_{u_{\|}} f_{\alpha 0} .
\end{aligned}
$$

Here we have omitted the details of the derivation for the sake of brevity, since they can be obtained from a previous publication [5]. In these expressions $f_{\alpha 0}$ is the distribution function for electrons of population $\alpha$, with the summation carried out over all electron populations. The ions do not appear explicitly since Eq. (1) has been developed for the case of high frequency oscillations. It is assumed that the ion distribution is effective as a neutralizing background and carries the current in the $y$ direction which must exist in the plasma in order to satisfy the equilibrium configuration with the $x$-dependent magnetic field pointing in the $z$ direction [5]. $u_{\perp}$ and $u_{\|}$are the perpendicular and parallel components of the normalized momentum, $\mathbf{u}=\mathbf{p} /\left(m_{\alpha} c\right), \gamma=\left(1+u^{2}\right)^{1 / 2}$, $J_{n}(z)$ and $J_{n}^{\prime}(z)$ are the Bessel functions of order $n$ and its derivative, and $\hat{\boldsymbol{e}}_{x}, \hat{\boldsymbol{e}}_{y}$, and $\hat{\boldsymbol{e}}_{z}$ are, respectively, the unit vectors in the $x, y$, and $z$ directions.

The geometry utilized in the derivation of Eq. (1) has been the following. The magnetic field has been considered pointing in the $z$ direction, and inhomogeneous in the $x$ direction, $\mathbf{B}_{0}=B_{0}(x) \hat{\boldsymbol{e}}_{z}$. The waves were assumed propagating in arbitrary directions, with $k_{\|}$and $k_{\perp}$ as the components of the wave vector, respectively, parallel and perpendicular to the magnetic field. The wave angular frequency has been denoted as $\omega . \psi$ denotes the angle between the vector $\mathbf{k}_{\perp}$ and the direction of the inhomogeneity. The inhomogeneity has been assumed to be weak, such that the cyclotron frequency has been written as

$$
\Omega_{\alpha}\left(x_{\alpha}^{\prime}\right) \sim \Omega_{\alpha}(x)\left[1+\epsilon\left(x_{\alpha}^{\prime}-x\right)\right],
$$

where $\Omega_{\alpha}(x)=\left[q_{\alpha} B_{0}(x) / m_{\alpha} c\right]$ is the particle cyclotron angular frequency, $m_{\alpha}$ is the particle rest mass, $q_{\alpha}$ is the particle charge, $c$ is the velocity of light, $\epsilon \equiv\left[\left(1 / B_{0}\right)\left(d B_{0}\right.\right.$ $\left.\left./ d x_{\alpha}^{\prime}\right)\right]_{x_{\alpha}^{\prime}=x}$, and $x_{\alpha}^{\prime}$ is the unperturbed position of particle $\alpha$.

The validity of the expressions given by Eq. (1) is limited to the case where the distribution function is azimuthally symmetric, $f_{\alpha 0}=f_{\alpha 0}\left(u_{\perp}^{2}, u_{\|}\right)$. Among the reasons for the choice of this distribution is the fact that it allows the emphasis on the study of magnetic field inhomogeneities and simplifies all other features of the problem. Other kinds of inhomogeneities, where the distribution is explicitly dependent on position and therefore must exhibit the dependence on the constant of motion $\bar{P}_{\alpha}=p_{\perp} \sin \varphi+m_{\alpha} \int{ }^{x} \Omega_{\alpha}\left(x^{\prime}\right) d x^{\prime}$, were already discussed in previous studies [2-4] $(\varphi$ is the azimuthal angle of $\mathbf{p}$ ).

The form of the effective dielectric tensor as given by Eq. (1) is not the most suitable for our present purposes. In order to rewrite Eq. (1) in a more convenient form, we first note that

$$
\begin{aligned}
e^{-i n \psi_{n \alpha}} & =\left[\frac{1-\left(\epsilon_{n} / k_{\perp}\right)^{2}-i 2 S_{n} \epsilon_{n} \sin (\psi) / k_{\perp}}{\xi_{n}^{+} \xi_{n}^{-}}\right]^{|n|} \\
& =\left[\frac{b_{\alpha}^{2}-\mathcal{K}_{n}^{2} \tau^{2}-i 2 S_{n} b_{\alpha} \sin (\psi) \mathcal{K}_{n} \tau}{W_{n}^{+} W_{n}^{-}}\right]^{|n|} \equiv\left(\frac{F_{n \alpha}(\tau)}{W_{n}^{+} W_{n}^{-}}\right)^{|n|},
\end{aligned}
$$

where

$$
\begin{gathered}
S_{n}=\operatorname{sgn}(n), \\
\mathcal{K}_{n}=n \epsilon c u_{\perp} / 2 \\
W_{n}^{ \pm}=\left[b_{\alpha}^{2} \pm 2 b_{\alpha} \cos (\psi) \mathcal{K}_{n} \tau+\mathcal{K}_{n}^{2} \tau^{2}\right]^{1 / 2} .
\end{gathered}
$$

Due to the dyadic $\boldsymbol{\Pi}_{n \alpha}^{-} \boldsymbol{\Pi}_{n \alpha}^{+}$the products involving Bessel functions of order $n$ and its derivatives are equal to the same products involving Bessel functions and derivatives of order $|n|$. Moreover, the derivatives of the Bessel functions appearing in Eq. (1) can be eliminated by the use of the wellknown recurrence relation

$$
J_{n}^{\prime}(z)=\frac{n}{z} J_{n}(z)-J_{n+1}(z)
$$

Therefore the effective dielectric tensor can be written as

$$
\begin{aligned}
& \overleftrightarrow{\varepsilon}=\overleftrightarrow{1}-i \sum_{\alpha} \frac{4 \pi q_{\alpha}^{2}}{m_{\alpha} \omega_{n \rightarrow-\infty}} \sum_{0}^{\infty} d \tau \\
& \times \int d^{3} u u_{\perp} \mathcal{L}\left(f_{\alpha 0}\right) e^{i D_{n \alpha} \tau}\left[F_{n \alpha}(\tau)\right]^{(|n|-1)} \frac{\boldsymbol{\Pi}_{n \alpha}^{-} \boldsymbol{\Pi}_{n \alpha}^{+}}{\left(W_{n}^{-} W_{n}^{+}\right)^{|n|}} \\
& -\hat{\boldsymbol{e}}_{z} \hat{\boldsymbol{e}}_{z} \sum_{\alpha} \frac{4 \pi q_{\alpha}^{2}}{m_{\alpha} \omega^{2}} \int d^{3} u \frac{u_{\|}}{\gamma} L\left(f_{\alpha 0}\right)\left(u_{\perp}^{2}, u_{\|}\right),
\end{aligned}
$$

where 


$$
\begin{gathered}
\boldsymbol{\Pi}_{n \alpha}^{ \pm}= \pm\left[n J_{|n|}\left(W_{n}^{ \pm}\right) G_{n \alpha}^{ \pm}(\tau)+i \frac{J_{|n|+1}\left(W_{n}^{ \pm}\right)}{W_{n}^{ \pm}} F_{n \alpha}^{1 / 2}(\tau) b_{\alpha} \sin \psi\right] \hat{\boldsymbol{e}}_{x} \\
+i\left[|n| J_{|n|}\left(W_{n}^{ \pm}\right) G_{n \alpha}^{ \pm}(\tau) \mp \frac{J_{|n|+1}\left(W_{n}^{ \pm}\right)}{W_{n}^{ \pm}} F_{n \alpha}^{1 / 2}(\tau)\left(b_{\alpha} \cos \psi \pm \mathcal{K}_{n} \tau\right)\right] \hat{\boldsymbol{e}}_{y}+\frac{u_{\|}}{u_{\perp}} J_{|n|}\left(W_{n}^{ \pm}\right) F_{n \alpha}^{1 / 2}(\tau) \hat{\boldsymbol{e}}_{z}, \\
G_{n \alpha}^{ \pm}(\tau)=i \sqrt{\frac{\mathcal{K}_{n} \tau \mp b_{\alpha} \cos \psi+i S_{n} b_{\alpha} \sin \psi}{\mathcal{K}_{n} \tau \pm b_{\alpha} \cos \psi+i S_{n} b_{\alpha} \sin \psi}}, \\
G_{n \alpha}^{+} G_{n \alpha}^{-}=-1, \quad F_{n \alpha}^{1 / 2} G_{n \alpha}^{ \pm}=-\left(\mathcal{K}_{n} \tau \mp b_{\alpha} \cos \psi+i S_{n} b_{\alpha} \sin \psi\right) .
\end{gathered}
$$

This expression looks as complex as the original, Eq. (1), but is is more appropriate to be used with the generalized loss-cone distribution function to be introduced in the following section.

\section{EFFECTIVE DIELECTRIC TENSOR FOR NONRELATIVISTIC DGH DISTRIBUTIONS}

The momentum integrals of Eq. (3) will now be evaluated by assuming that the particles of population $\alpha$ have a twotemperature nonrelativistic DGH distribution function

$$
f_{\alpha 0}\left(u_{\perp}^{2}, u_{\|}\right)=n_{\alpha}\left(\frac{\mu_{\| \alpha}}{2 \pi}\right)^{1 / 2} \frac{\mu_{\perp \alpha}}{2 \pi} \frac{\mu_{\perp \alpha}^{l_{\alpha}}}{2^{l_{\alpha}} l_{\alpha} !} u_{\perp}^{2 l_{\alpha}} e^{-\left(\mu_{\perp \alpha} u_{\perp}^{2}+\mu_{\| \alpha} u_{\|}^{2}\right) / 2},
$$

where $n_{\alpha}$ is the density of the species $\alpha, l_{\alpha}$ is an integer quantity known as "the loss-cone index," $\mu_{\perp \alpha}=m_{\alpha} c^{2} / T_{\perp \alpha}$, and $\mu_{\| \alpha}=m_{\alpha} c^{2} / T_{\| \alpha}$. The ratio between perpendicular and parallel temperatures will be denoted as $r_{T}=T_{\perp \alpha} / T_{\| \alpha}$.

Introducing the distribution (4) into Eq. (3), the integrations in the $u$ space can be performed. The calculations are made with use of the weakly relativistic approximation, a reasonable assumption for $T_{\alpha} \lesssim 10 \mathrm{keV}$, which implies that $\gamma \approx 1+u^{2} / 2$. The nonrelativistic distribution function given by Eq. (4) is consistent with this weakly relativistic approximation, and it is frequently employed for the description of oblique absorption of cyclotron radiation in mild temperature loss-cone plasmas. As a result of these integrations in momentum space, the components of the dielectric tensor for the particular case of $l_{\alpha}=0$ (anisotropic Maxwellian distribution) are written in terms of a function denoted by $\mathcal{G}_{r, q, p, m, l}$, hereafter denominated "inhomogeneous plasma dispersion function" and defined as follows:

$$
\mathcal{G}_{r, q, p, m, l}\left(z, \beta, \sigma_{\alpha}, \nu_{\alpha}, \chi_{n \alpha}, r_{T}\right) \equiv-i \int_{0}^{\infty} d t \frac{(i t)^{r} e^{i z t} e^{-\beta t^{2} /(1-i t)}}{(1-i t)^{q}\left(1-i r_{T} \sigma_{\alpha} t\right)^{p}} e^{-r_{T}\left(\nu_{\alpha}^{2}+\chi_{n t^{t}}^{2}\right) /\left(1-i r_{T} \sigma_{\alpha} t\right)} \frac{\left[H_{n \alpha}(t)\right]^{m}}{\left[S_{n \alpha}(t)\right]^{l}} I_{l}\left(\frac{S_{n \alpha}(t)}{1-i r_{T} \sigma_{\alpha} t}\right),
$$

where

$$
H_{n \alpha}(t)=\nu_{\alpha}^{2}-i 2 S_{n} \nu_{\alpha} \sin (\psi) \chi_{n \alpha} t-\chi_{n \alpha}^{2} t^{2}, \quad S_{n \alpha}(t)=\sqrt{\nu_{\alpha}^{4}-2 \nu_{\alpha}^{2} \cos (2 \psi) \chi_{n \alpha}^{2} t^{2}+\chi_{n \alpha}^{4} t^{4}},
$$

and where we have introduced the following definitions: $t=\left(\omega / \mu_{\alpha}\right) \tau, z=\mu_{\alpha} \delta_{n \alpha}, \delta_{n \alpha}=1-n Y_{\alpha}(1+\epsilon x), \beta=\mu_{\alpha} N_{\|}^{2} / 2$, $\chi_{n \alpha}=\mu_{\alpha}^{1 / 2} \rho_{n}, \sigma_{\alpha}=1-N_{B} N_{\perp}(\sin \psi) / Y_{\alpha}, \nu_{\alpha}=N_{\perp} / \mu_{\alpha}^{1 / 2} Y_{\alpha}, Y_{\alpha}=\Omega_{\alpha} / \omega, \rho_{n}=n N_{B} / 2$, and $N_{B}=\epsilon c / \omega . I_{n}(z)$ is the modified Bessel function of the first kind. Details of the calculation are shown in Appendix A, where for simplicity we only consider the isotropic case $\left(T_{\perp}=T_{\|}\right)$.

For the more general case of $l_{\alpha} \neq 0$, the calculations are similar, although tedious, and the outcome is

$$
\begin{aligned}
\varepsilon_{11}= & 1-\sum_{\alpha} \mu_{\perp \alpha} X_{\alpha} \frac{(-1)^{l \alpha}}{l_{\alpha} !}\left\{\Delta ^ { ( 1 ) } \sum _ { n \rightarrow - \infty } ^ { \infty } \left[n^{2} \mathcal{G}_{0,1 / 2,1,|n|-1,|n|}^{*}+2 n r_{T} \nu_{\alpha} \sin (\psi) \chi_{n \alpha}\left(\mathcal{G}_{1,1 / 2,2,|n|-1,|n|}^{*}+\mathcal{G}_{1,1 / 2,2,|n|,|n|+1}^{*}\right)\right.\right. \\
& \left.\left.+2 r_{T}^{2} \nu_{\alpha}^{2} \sin ^{2} \psi\left(\mathcal{G}_{0,1 / 2,3,|n|,|n|}^{*}-\frac{|n|}{r_{T}} \mathcal{G}_{0,1 / 2,2,|n|-1,|n|}^{*}-\nu_{\alpha}^{2} \mathcal{G}_{0,1 / 2,3,|n|,|n|+1}^{*}+\chi_{n \alpha}^{2} \mathcal{G}_{2,1 / 2,3,|n|,|n|+1}^{*}\right)\right]\right\}_{h=0} \\
\varepsilon_{22}= & 1-\sum_{\alpha} \mu_{\perp \alpha} X_{\alpha} \frac{(-1)^{l} \alpha}{l_{\alpha} !}\left\{\Delta ^ { ( 1 ) } \sum _ { n \rightarrow - \infty } ^ { \infty } \left[n^{2} \mathcal{G}_{0,1 / 2,1,|n|-1,|n|}^{*}-2 n r_{T} \nu_{\alpha} \sin (\psi) \chi_{n \alpha}\left(\mathcal{G}_{1,1 / 2,2,|n|-1,|n|}^{*}-\mathcal{G}_{1,1 / 2,2,|n|,|n|+1}^{*}\right)\right.\right. \\
& +2 r_{T}^{2} \nu_{\alpha}^{2} \cos ^{2} \psi\left(\mathcal{G}_{0,1 / 2,3,|n|,|n|}^{*}-\frac{|n|}{r_{T}} \mathcal{G}_{0,1 / 2,2,|n|-1,|n|}^{*}-\nu_{\alpha}^{2} \mathcal{G}_{0,1 / 2,3,|n|,|n|+1}^{*}+\chi_{n \alpha}^{2} \mathcal{G}_{2,1 / 2,3,|n|,|n|+1}^{*}\right) \\
& \left.\left.+2 r_{T}^{2} \chi_{n \alpha}^{2}\left(\mathcal{G}_{2,1 / 2,3,|n|,|n|}^{*}+\frac{|n|}{r_{T}} \mathcal{G}_{2,1 / 2,2,|n|-1,|n|}^{*}-\nu_{\alpha}^{2} \mathcal{G}_{2,1 / 2,3,|n|,|n|+1}^{*}+\chi_{n \alpha}^{2} \mathcal{G}_{4,1 / 2,3,|n|,|n|+1}^{*}\right)\right]\right\}_{h=0},
\end{aligned}
$$




$$
\begin{aligned}
& \varepsilon_{33}=1-\sum_{\alpha} X_{\alpha}\left[1-\delta l_{\alpha, 0} / r_{T}\right]-\sum_{\alpha} \mu_{\perp \alpha} X_{\alpha} \frac{(-1)^{l_{\alpha}}}{l_{\alpha} !}\left\{\Delta^{(3)} \sum_{n \rightarrow-\infty}^{\infty}\left[\mathcal{G}_{0,3 / 2,1,|n|,|n|}^{*}\right\}_{h=0},\right. \\
& \boldsymbol{\varepsilon}_{\left(\begin{array}{ll}
12 \\
{ }_{12}
\end{array}\right)}=-\sum_{\alpha} \mu_{\perp \alpha} X_{\alpha} \frac{(-1)^{l} \alpha}{l_{\alpha} !}\left\{\Delta ^ { ( 1 ) } \sum _ { n \rightarrow - \infty } ^ { \infty } \left[ \pm i 2 r_{T}^{2} \nu_{\alpha} \sin (\psi) \chi_{n \alpha}\left(\mathcal{G}_{1,1 / 2,3,|n|,|n|}^{*}-\nu_{\alpha}^{2} \mathcal{G}_{1,1 / 2,3,|n|,|n|+1}^{*}+\chi_{n \alpha}^{2} \mathcal{G}_{3,1 / 2,3,|n|,|n|+1}^{*}\right)\right.\right. \\
& \pm i n r_{T}\left(\frac{|n|}{r_{T}} \mathcal{G}_{0,1 / 2,1,|n|-1,|n|}^{*}-\nu_{\alpha}^{2}\left(\mathcal{G}_{0,1 / 2,2,|n|-1,|n|}^{*}-\mathcal{G}_{0,1 / 2,2,|n|,|n|+1}^{*}\right)+\chi_{n \alpha}^{2}\left(\mathcal{G}_{2,1 / 2,2,|n|-1,|n|}^{*}+\mathcal{G}_{2,1 / 2,2,|n|,|n|+1}^{*}\right)\right)-r_{T}^{2} \nu_{\alpha}^{2} \sin (2 \psi) \\
& \left.\left.\times\left(\mathcal{G}_{0,1 / 2,3,|n|,|n|}^{*}-\frac{|n|}{r_{T}} \mathcal{G}_{0,1 / 2,2,|n|-1,|n|}^{*}-\nu_{\alpha}^{2} \mathcal{G}_{0,1 / 2,3,|n|,|n|+1}^{*}+\chi_{n \alpha}^{2} \mathcal{G}_{2,1 / 2,3,|n|,|n|+1}^{*}\right)-2 n r_{T} \nu_{\alpha} \cos (\psi) \chi_{n \alpha} \mathcal{G}_{1,1 / 2,2,|n|-1,|n|}^{*}\right]\right\}_{h=0}, \\
& \varepsilon_{\left(\begin{array}{l}
13 \\
31
\end{array}\right)}= \pm i N_{\|} \sum_{\alpha} \mu_{\perp \alpha} \mu_{\| \alpha}^{1 / 2} X_{\alpha} \frac{(-1)^{l_{\alpha}}}{l_{\alpha} !}\left\{\Delta ^ { ( 2 ) } \sum _ { n \rightarrow - \infty } ^ { \infty } \left\{n\left(\chi_{n \alpha} \mathcal{G}_{1,3 / 2,1,|n|-1,|n|}^{*} \pm i \nu_{\alpha} e^{ \pm i S_{n} \psi} \mathcal{G}_{0,3 / 2,1,|n|-1,|n|}^{*}\right)\right.\right. \\
& \left.\left.+r_{T} \nu_{\alpha}(\sin \psi)\left[\mathcal{G}_{0,3 / 2,2,|n|,|n|}^{*}-\nu_{\alpha}^{2} \mathcal{G}_{0,3 / 2,2,|n|,|n|+1}^{*} \pm i 2 \nu_{\alpha} \cos (\psi) \chi_{n \alpha} \mathcal{G}_{1,3 / 2,2,|n|,|n|+1}^{*}+\chi_{n \alpha}^{2} \mathcal{G}_{2,3 / 2,2,|n|,|n|+1}^{*}\right]\right\}\right\}_{h=0}, \\
& \varepsilon_{\left(\begin{array}{l}
23 \\
32
\end{array}\right)}=N_{\|} \sum_{\alpha} \mu_{\perp \alpha} \mu_{\| \alpha}^{1 / 2} X_{\alpha} \frac{(-1)^{l} \alpha}{l_{\alpha} !}\left\{\Delta ^ { ( 2 ) } \sum _ { n \rightarrow - \infty } ^ { \infty } \left[|n|\left(\chi_{n \alpha} \mathcal{G}_{1,3 / 2,1,|n|-1,|n|}^{*} \pm i \nu_{\alpha} e^{ \pm i S_{n} \psi} \mathcal{G}_{0,3 / 2,1,|n|-1,|n|}^{*}\right)\right.\right. \\
& +r_{T} \nu_{\alpha}^{2} \cos (2 \psi) \chi_{n \alpha} \mathcal{G}_{1,3 / 2,2,|n|,|n|+1}^{*}+r_{T} \chi_{n \alpha}\left(\mathcal{G}_{1,3 / 2,2,|n|,|n|}^{*}+\chi_{n \alpha}^{2} \mathcal{G}_{3,3 / 2,2,|n|,|n|+1}^{*}\right) \\
& \left.\left.\mp i r_{T} \nu_{\alpha} \cos \psi\left(\mathcal{G}_{0,3 / 2,2,|n|,|n|}^{*}-\nu_{\alpha}^{2} \mathcal{G}_{0,3 / 2,2,|n|,|n|+1}^{*}-\chi_{n \alpha}^{2} \mathcal{G}_{2,3 / 2,2,|n|,|n|+1}^{*}\right)\right]\right\}_{h=0} .
\end{aligned}
$$

The function denoted by $\mathcal{G}_{r, q, p, m, l}^{*}$ is defined

$$
\begin{aligned}
& \mathcal{G}_{r, q, p, m, l}^{*}\left(z, \beta, \sigma_{\alpha}, \nu_{\alpha}, \chi_{n \alpha}, r_{T} ; h\right) \\
& \quad \equiv-i \int_{0}^{\infty} d t \frac{(i t)^{r} e^{i z t} e^{-\beta t^{2} /(1-i t)}}{(1-i t)^{q}\left(1-i r_{T} \sigma_{\alpha} t+h\right)^{p}} e^{-r_{T}\left(\nu_{\alpha}^{2}+\chi_{n \alpha}^{2} t^{2}\right) /\left(1-i r_{T} \sigma_{\alpha} t+h\right)} \frac{\left[H_{n \alpha}(t)\right]^{m}}{\left[S_{n \alpha}(t)\right]^{l}} I_{l}\left(\frac{S_{n \alpha}(t)}{1-i r_{T} \sigma_{\alpha} t+h}\right) .
\end{aligned}
$$

The differential operators $\Delta^{(n)}$ are given by

$$
\begin{aligned}
& \Delta^{(1)} \equiv\left[1+\left(1-r_{T}\right) N_{\|}^{2} \Delta_{1,1}\right] \frac{\partial^{l_{\alpha}}}{\partial h^{l_{\alpha}}}+l_{\alpha}\left(1+N_{\|}^{2} \Delta_{1,1}\right) \frac{\partial^{\left(l_{\alpha}-1\right)}}{\partial h^{\left(l_{\alpha}-1\right)}}, \\
& \Delta^{(2)} \equiv\left[\Delta_{1,0}+\frac{1-r_{T}}{\mu_{\| \alpha}}\left(1+\mu_{\| \alpha} N_{\|}^{2} \Delta_{2,1}\right)\right] \frac{\partial^{l_{\alpha}}}{\partial h^{l_{\alpha}}}+l_{\alpha}\left[\Delta_{1,0}+\frac{1}{\mu_{\| \alpha}}\left(1+\mu_{\| \alpha} N_{\|}^{2} \Delta_{2,1}\right)\right] \frac{\partial^{\left(l_{\alpha}-1\right)}}{\partial h^{\left(l_{\alpha}-1\right)}} \\
& \Delta^{(3)} \equiv\left[1+\mu_{\| \alpha} N_{\|}^{2} \Delta_{2,1}+\left(1-r_{T}\right) N_{\|}^{2}\left(3 \Delta_{1,1}+\mu_{\| \alpha} N_{\|}^{2} \Delta_{3,2}\right)\right] \frac{\partial^{l_{\alpha}}}{\partial h^{l_{\alpha}}}+l_{\alpha}\left[1+\mu_{\| \alpha} N_{\|}^{2} \Delta_{2,1}+N_{\|}^{2}\left(3 \Delta_{1,1}+\mu_{\| \alpha} N_{\|}^{2} \Delta_{3,2}\right)\right] \frac{\partial^{\left(l_{\alpha}-1\right)}}{\partial h^{\left(l_{\alpha}-1\right)}},
\end{aligned}
$$

where $\Delta_{r^{\prime}, q^{\prime}} \mathcal{G}_{r, q, p, m, l}^{*} \equiv \mathcal{G}_{\left(r+r^{\prime}\right),\left(q+q^{\prime}\right), p, m, l}^{*}$.

The $\mathcal{G}_{r, q, p, m, l}^{*}$ function may be regarded simply as an auxiliary function appearing for loss-cone plasmas, since after application of the differential operators $\Delta^{(n)}$, the outcome can again be written using the function $\mathcal{G}_{r, q, p, m, l}^{*}$, evaluated for $h=0$, which is simply the inhomogeneous plasma dispersion function defined by Eq. (5),

$$
\begin{gathered}
\left.\mathcal{G}_{r, q, p, m, l}^{*}\left(z, \beta, \sigma_{\alpha}, \nu_{\alpha}, \chi_{n \alpha}, r_{T} ; h\right)\right|_{h=0} \\
\quad=\mathcal{G}_{r, q, p, m, l}\left(z, \beta, \sigma_{\alpha}, \nu_{\alpha}, \chi_{n \alpha}, r_{T}\right) .
\end{gathered}
$$

Therefore the inhomogeneous plasma dispersion function given by Eq. (5) plays a central role in the present formulation. In Sec. IV we present some properties and special expansions of this function which show its relationships with other plasma dispersion functions already known from the study of homogeneous plasmas. In Appendix B some of these properties are utilized in order to show that the antiHermitian part of the effective dielectric tensor is free of nonresonant terms, originating an absorption coefficient which really describes absorption and/or amplification due to the wave-particle interaction. 


\section{THE INHOMOGENEOUS PLASMA DISPERSION FUNCTION AND ITS RELATIONSHIP TO OTHER DISPERSION FUNCTIONS}

The inhomogeneous plasma dispersion function is defined by an integral expression and can be related to other wellknown plasma dispersion functions (PDF) appearing in the case of homogeneous plasmas. This feature is particularly useful for computational reasons, since the analytic properties of these functions are well studied, and many useful mathematical relationships can be found in the literature. In what follows, we will initially consider some particular cases, which can be dealt with more easily, before discussing the most general direction of propagation in inhomogeneous cases.

\section{A. Homogeneous case $(\epsilon=0)$}

In the homogeneous case, Eq. (5) reduces to

$$
\begin{aligned}
\mathcal{G}_{r, q, p, m, l}= & -i \nu_{\alpha}^{2(m-l)} \int_{0}^{\infty} d t \frac{(i t)^{r} e^{i z t}}{(1-i t)^{q+p}} \\
& \times e^{-\beta t^{2} /(1-i t)} e^{-\nu_{\alpha}^{2} /(1-i t)} I_{l}\left(\frac{\nu_{\alpha}^{2}}{1-i t}\right) .
\end{aligned}
$$

This equation can be written in familiar form as

$$
\mathcal{G}_{r, q, p, m, l}=\nu_{\alpha}^{2(m-l)} \mathcal{R}_{q+p, r}\left(z, \beta, \nu_{\alpha}^{2}, l\right),
$$

where $\mathcal{R}_{l, m}$ is the PDF defined by Eq. (62) of Ref. [7]. This is the weakly relativistic PDF valid for waves propagating with arbitrary perpendicular wave number in magnetized thermal plasmas [7]. It can be related to Shkarofsky and Dnestrovskii functions in the limit of nearly perpendicular propagation, and all its properties can be found in Refs. $[7,8]$.

\section{B. Waves propagating with $\mathbf{k}_{\perp}$ parallel to the inhomogeneity $(\psi=0)$}

In the case of $\psi=0$ the functions $H_{n \alpha}(t)$ and $S_{n \alpha}(t)$ appearing in the integrand of Eq. (5) are greatly simplified, and the inhomogeneous PDF reduces to

$$
\begin{aligned}
\mathcal{G}_{r, q, p, m, l}= & -i \int_{0}^{\infty} d t \frac{(i t)^{r} e^{i z t}}{(1-i t)^{q+p}} e^{-\left[\nu_{\alpha}^{2}+\left(\beta+\chi_{n \alpha}^{2}\right) t^{2}\right] /(1-i t)} \\
& \times\left(\nu_{\alpha}^{2}-\chi_{n \alpha}^{2} t^{2}\right)^{m-l} I_{l}\left(\frac{\nu_{\alpha}^{2}-\chi_{n \alpha}^{2} t^{2}}{1-i t}\right)
\end{aligned}
$$

This expression can be further modified by the use of an integral representation of the modified Bessel function [9]:

$$
I_{n}(z)=\frac{z^{n}}{(2 n-1) ! ! \pi} \int_{-1}^{1} d y\left(1-y^{2}\right)^{n-1 / 2} e^{ \pm z y}
$$

resulting in the expression

$$
\mathcal{G}_{r, q, p, m, l}=\sum_{s=0}^{m}\left(\begin{array}{c}
m \\
s
\end{array}\right) \frac{\chi_{n \alpha}^{2 s} \nu_{\alpha}^{2(m-s)}}{\pi(2 l-1) ! !} \int_{-1}^{1} d y\left(1-y^{2}\right)^{l-1 / 2} e^{-\nu_{\alpha}^{2}(1-y)} \mathcal{F}_{(q+p+l),(r+2 s)}\left(z-\nu_{\alpha}^{2}(1-y), \beta+\chi_{n \alpha}^{2}(1+y)-\nu_{\alpha}^{2}(1-y)\right)
$$

where

$$
\mathcal{F}_{q, r}(z, a)=-i \int_{0}^{\infty} d t \frac{(i t)^{r} e^{i z t}}{(1-i t)^{q}} e^{-a t^{2} /(1-i t)}=-i e^{-a} \int_{0}^{\infty} d t \frac{(i t)^{r} e^{i(z-a) t}}{(1-i t)^{q}} e^{a /(1-i t)}
$$

is the well-known Shkarofsky function $[7,8]$.

Although complicated, Eq. (13) can be simplified when the particle Larmor radius is small $\left(\left|\nu_{\alpha}^{2}\right| \ll 1\right.$.) With this assumption we can expand the term $\exp \left[-\nu_{\alpha}^{2}(1-y) /(1-i t)\right]$ in powers of $\nu_{\alpha}^{2}$, retaining only the first two terms of the expansion. Inserting this expansion in Eq. (13) and retaining only the lowest order terms on the ensuing summation, we arrive at

$$
\begin{aligned}
\mathcal{G}_{r, q, p, m, l}= & \frac{1}{\pi(2 l-1) ! !}\left[\chi_{n \alpha}^{2 m}\left(\int_{-1}^{1} d y\left(1-y^{2}\right)^{l-1 / 2} \mathcal{F}_{q+p+l, r+2 m}-\nu_{\alpha}^{2} \int_{-1}^{1} d y\left(1-y^{2}\right)^{l-1 / 2}(1-y) \mathcal{F}_{q+p+l+1, r+2 m}\right)\right. \\
& \left.+m \nu_{\alpha}^{2} \chi_{n \alpha}^{2(m-1)} \int_{-1}^{1} d y\left(1-y^{2}\right)^{l-1 / 2} \mathcal{F}_{q+p+l, r+2 m-2}\right]
\end{aligned}
$$

in which the Shkarofsky function no longer depends on $\nu_{\alpha}^{2}, \mathcal{F}_{q, r}=\mathcal{F}_{q, r}\left(z, \beta+\chi_{n \alpha}^{2}(1+y)\right)$.

Expression (14), when used in conjunction with the assumption of small $\chi_{n \alpha}^{2}$, leads to greater simplification on the dielectric tensor components. It will be used in Sec. $\mathrm{V}$ to derive a very simple expression for the dispersion relation of the ordinary mode.

Similar approximations can be made for the general case $\psi \neq 0$, which will be considered in the following sections. However, such approximations will not be explicitly shown, since they would not be useful for the example appearing in Sec. V. 


\section{Waves propagating with $k_{\perp}$ perpendicular to the inhomogeneity $(\psi=\pi / 2)$}

In the case of waves propagating with $\mathbf{k}_{\perp}$ perpendicular to the inhomogeneity the functions $H_{n \alpha}(t)$ and $S_{n \alpha}(t)$ also can be reduced to simple expressions, and the inhomogeneous PDF can be written as

$$
\mathcal{G}_{r, q, p, m, l}=-i \int_{0}^{\infty} d t \frac{(i t)^{r} e^{i z t} e^{-\beta t^{2} /(1-i t)}}{(1-i t)^{q}\left(1-i \sigma_{\alpha} t\right)^{p}} e^{-\left(\nu_{\alpha}^{2}+\chi_{n \alpha}^{2} t^{2}\right) /\left(1-i \sigma_{\alpha} t\right)} \frac{\left(\nu_{\alpha}-i S_{n} \chi_{n \alpha} t\right)^{2 m}}{\left(\nu_{\alpha}^{2}+\chi_{n \alpha}^{2} t^{2}\right)^{l}} I_{l}\left(\frac{\nu_{\alpha}^{2}+\chi_{n \alpha}^{2} t^{2}}{1-i \sigma_{\alpha} t}\right) .
$$

This expression for the inhomogeneous plasma dispersion function can also be written in terms of Shkarofsky functions, under some restrictive conditions. We will demonstrate this by considering the following.

In the integrand of Eq. (15) we have an exponential function which can be written as follows, after introducing the representation given by Eq. (12):

$$
\exp \left[-\frac{\nu_{\alpha}^{2}+\chi_{n \alpha}^{2} t^{2}}{1-i \sigma_{\alpha} t}(1+y)\right]=\exp \left[-\frac{\nu_{\alpha}^{2}+\chi_{n \alpha}^{2} t^{2}}{\sigma_{\alpha}(1-i t)}(1+y)\right] \sum_{s=0}^{\infty} \frac{1}{s !}\left[\frac{\left(\sigma_{\alpha}^{-1}-1\right)\left(\nu_{\alpha}^{2}+\chi_{n \alpha}^{2} t^{2}\right)(1+y)}{(1-i t)\left(1-i \sigma_{\alpha} t\right)}\right]^{s} .
$$

This series representation remains finite even for $t \rightarrow \infty$. In its derivation we have used the following identity:

$$
\frac{1}{1-i \sigma_{\alpha} t}=\frac{1}{1-i t}\left(\frac{1}{\sigma_{\alpha}}+\frac{1-\sigma_{\alpha}^{-1}}{1-i \sigma_{\alpha} t}\right)
$$

With use of Eq. (16), the PDF is modified into

$$
\begin{aligned}
\mathcal{G}_{r, q, p, m, l}= & \frac{-i}{(2 l-1) ! ! \pi} \sum_{s=0}^{\infty} \frac{\left(\sigma_{\alpha}^{-1}-1\right)^{s}}{s !} \int_{-1}^{1} d y\left(1-y^{2}\right)^{l-1 / 2}(1+y)^{s} e^{-\nu_{\alpha}^{2}(1+y) / \sigma_{\alpha}} e^{-\Phi_{y}} \\
& \times \int_{0}^{\infty} d t \frac{(i t)^{r} e^{i\left(\Upsilon_{y}-\Phi_{y}\right) t} e^{\Phi_{y} /(1-i t)}}{(1-i t)^{q+s}\left(1-i \sigma_{\alpha} t\right)^{p+l+s}}\left(\nu_{\alpha}^{2}+\chi_{n \alpha}^{2} t^{2}\right)^{s}\left(\nu_{\alpha}-i S_{n} \chi_{n \alpha} t\right)^{2 m},
\end{aligned}
$$

where

$$
\Phi_{y} \equiv \beta+\left(\chi_{n \alpha}^{2}-\nu_{\alpha}^{2}\right)(1+y) / \sigma_{\alpha}, \quad \Upsilon_{y} \equiv z-\nu_{\alpha}^{2}(1+y) / \sigma_{\alpha}
$$

The quantities $\left(\nu_{\alpha}^{2}+\chi_{n \alpha}^{2} t^{2}\right)^{s}$ and $\left(\nu_{\alpha}-i S_{n} \chi_{n \alpha} t\right)^{2 m}$ appearing in the integrand of the PDF can be expanded in powers of $t$, and we obtain

$$
\begin{aligned}
\mathcal{G}_{r, q, p, m, l}= & \frac{1}{(2 l-1) ! ! \pi} \sum_{s=0}^{\infty} \frac{\left(\sigma_{\alpha}^{-1}-1\right)^{s}}{s !} \sum_{k=0}^{2 m} \sum_{h=0}^{s}\left(\begin{array}{c}
2 m \\
k
\end{array}\right)\left(\begin{array}{c}
s \\
h
\end{array}\right)(-1)^{h+k} \nu_{\alpha}^{2(m+s)-h-k}\left(S_{n} \chi_{n \alpha}\right)^{2 h+k} \\
& \times \int_{-1}^{1} d y\left(1-y^{2}\right)^{l-1 / 2}(1+y)^{s} e^{-\nu_{\alpha}^{2}(1+y) / \sigma_{\alpha}} V_{(r+2 h+k),(q+s),(p+l+s)}\left(\Upsilon_{y}, \Phi_{y} ; \sigma_{\alpha}\right),
\end{aligned}
$$

where

$$
V_{r, q, p}\left(z, a ; \sigma_{\alpha}\right)=\frac{\partial^{r}}{\partial z^{r}} W_{q, p}\left(z, a ; \sigma_{\alpha}\right), \quad W_{q, p}\left(z, a ; \sigma_{\alpha}\right)=-i \int_{0}^{\infty} d t \frac{e^{i z t} e^{-a t^{2} /(1-i t)}}{(1-i t)^{q}\left(1-i \sigma_{\alpha} t\right)^{p}}
$$

The function $W_{q, p}\left(z, a ; \sigma_{\alpha}\right)$ has been studied in Refs. [10,11], appearing in the case of a homogeneous plasma with a Maxwellian distribution featuring temperature anisotropy. In that case, the parameter $\sigma_{\alpha}$ is related to the temperature anisotropy. In our case, it is the inhomogeneity that plays the role of the anisotropy. Equation (17) can be written in terms of Shkarofsky functions in particular cases [10]. When $\left|\sigma_{\alpha}^{-1}-1\right|<1$, the "'anisotropic', term appearing in the denominator of Eq. (17) can be expanded as

$$
\frac{1}{\left(1-i \sigma_{\alpha} t\right)^{p}}=\frac{1}{\sigma_{\alpha}^{p}} \sum_{j=0}^{\infty}\left(\begin{array}{c}
p+j-1 \\
p-1
\end{array}\right) \frac{\left(1-\sigma_{\alpha}^{-1}\right)^{j}}{(1-i t)^{p+j}}
$$

When this expansion is introduced into Eq. (17) we obtain

$$
V_{(r+2 h+k),(q+s),(p+l+s)}\left(\Phi_{y}, \Upsilon_{y} ; \sigma_{\alpha}\right)=\frac{1}{\sigma_{\alpha}^{p+l+s}} \sum_{j=0}^{\infty}\left(\begin{array}{c}
p+l+s+j-1 \\
p+l+s-1
\end{array}\right)\left(1-\sigma_{\alpha}^{-1}\right)^{j} \mathcal{F}_{(q+p+l+2 s+j),(r+k+2 h)}\left(\Upsilon_{y}, \Phi_{y}\right)
$$


which is the desired result. The validity condition for the use of this form of the PDF is not very restrictive. For instance, in present day tokamaks $\epsilon \approx 10^{-2} \mathrm{~cm}^{-1}, B \propto 10^{4} \mathrm{G},\left|\Omega_{e}\right| \propto 10^{11} \mathrm{~Hz}$, and the expansion utilized is valid as long as $\left|N_{\perp}\right| \lesssim 10^{2}$, which is frequently satisfied for electron cyclotron waves.

\section{Waves propagating at an arbitrary angle relative to the inhomogeneity}

We will now consider the case of electromagnetic waves propagating in arbitrary directions relatively to the inhomogeneity and to the magnetic field. Equation (5) can be modified by considering that the argument of the Bessel function can be considerably simplified by using Gegenbauer's addition theorem (Eq. 9.1.80 of Ref. [9])

$$
\frac{1}{S_{n \alpha}^{l}} I_{l}\left(\frac{S_{n \alpha}}{1-i \sigma_{\alpha} t}\right)=\frac{\left(1-i \sigma_{\alpha} t\right)^{l}}{\left(\nu_{\alpha}^{2} \chi_{n \alpha}^{2} t^{2}\right)^{l}} \sum_{k=0}^{\infty}(-1)^{k} A_{k}^{l} I_{l+k}\left(\frac{\nu_{\alpha}^{2}}{1-i \sigma_{\alpha} t}\right) I_{l+k}\left(\frac{\chi_{n \alpha}^{2} t^{2}}{1-i \sigma_{\alpha} t}\right),
$$

where

$$
\begin{gathered}
A_{k}^{l} \equiv 2^{l}(l-1) !(l+k) C_{k}^{(l)}(\cos 2 \psi), \quad C_{k}^{(l)}=\sum_{m=0}^{k}\left(\begin{array}{c}
l+m-1 \\
l-1
\end{array}\right)\left(\begin{array}{c}
l+k-m-1 \\
l-1
\end{array}\right) \cos [2(k-2 m) \psi], \\
C_{k}^{(l)}(1)=\left(\begin{array}{c}
k+2 l-1 \\
k
\end{array}\right) .
\end{gathered}
$$

This is a general expansion, which is useful for computational purposes only when a small Larmor radius approximation is valid.

With use of expressions (20) and (12), it is possible to write down the inhomogeneous PDF as

$$
\begin{aligned}
\mathcal{G}_{r, q, p, m, l}= & -i \sum_{k=0}^{\infty} \frac{A_{k}^{l} \chi_{n \alpha}^{2 k}}{[2(l+k)-1] ! ! \pi \nu_{\alpha}^{2 l}} \int_{-1}^{1} d y\left(1-y^{2}\right)^{l+k-1 / 2} \int_{0}^{\infty} d t \frac{(i t)^{r+2 k} e^{i z t} e^{-\beta t^{2} /(1-i t)}}{(1-i t)^{q}\left(1-i \sigma_{\alpha} t\right)^{p+k}} e^{-\chi_{n \alpha}^{2}(1+y) t^{2} /\left(1-i \sigma_{\alpha} t\right)} \\
& \times\left[H_{n \alpha}(t)\right]^{m} \mathcal{H}_{l+k}\left(\frac{\nu_{\alpha}^{2}}{1-i \sigma_{\alpha} t}\right),
\end{aligned}
$$

where

$$
\mathcal{H}_{n}(z) \equiv e^{-z} I_{n}(z)
$$

The function $H_{n \alpha}(t)$ can also be expanded, and we arrive at

$$
\begin{aligned}
\mathcal{G}_{r, q, p, m, l}= & \frac{1}{\pi} \sum_{k=0}^{\infty} \frac{A_{k}^{l}}{[2(l+k)-1] ! !} \sum_{\lambda=0}^{m} \sum_{\delta=0}^{m-\lambda}\left(\begin{array}{c}
m \\
\lambda
\end{array}\right)\left(\begin{array}{c}
m-\lambda \\
\delta
\end{array}\right)\left(-2 S_{n} \sin \psi\right)^{\lambda} \nu_{\alpha}^{2(m-l-\delta)-\lambda} \chi_{n \alpha}^{2(k+\delta)+\lambda} \\
& \times \int_{-1}^{1} d y\left(1-y^{2}\right)^{l+k-1 / 2} Q_{[r+2(k+\delta)+\lambda], q,(p+k),(l+k)}\left(z, \beta, \chi_{n \alpha}^{2}(1+y), \nu_{\alpha}, \sigma_{\alpha}\right),
\end{aligned}
$$

where

$$
Q_{r, q, p, l}\left(z, a, b, \nu_{\alpha}, \sigma_{\alpha}\right) \equiv-i \int_{0}^{\infty} d t \frac{(i t)^{r} e^{i z t} e^{-a t^{2} /(1-i t)}}{(1-i t)^{q}\left(1-i \sigma_{\alpha} t\right)^{p}} e^{-b t^{2} /\left(1-i \sigma_{\alpha} t\right)} \mathcal{H}_{l}\left(\frac{\nu_{\alpha}^{2}}{1-i \sigma_{\alpha} t}\right)
$$

Using the same procedure utilized in the derivation of Eq.

(16) results in the expression:

$$
\begin{aligned}
Q_{r, q, p, l}\left(z, a, b, \nu_{\alpha}, \sigma_{\alpha}\right) & \\
= & \sum_{s=0}^{\infty} \frac{b^{s}}{s !}\left(1-\sigma_{\alpha}^{-1}\right)^{s} \\
& \times P_{(r+2 s),(q+s),(p+s)}^{(l)}\left(z, a+b / \sigma_{\alpha}, \nu_{\alpha}^{2} ; \sigma_{\alpha}\right), \\
& \quad P_{r, q, p}^{(l)}\left(z, c, \nu_{\alpha}^{2} ; \sigma_{\alpha}\right)=\frac{\partial^{r}}{\partial z^{r}} R_{q, p}^{(l)}\left(z, c, \nu_{\alpha}^{2} ; \sigma_{\alpha}\right),
\end{aligned}
$$

$$
\begin{aligned}
R_{q, p}^{(l)}\left(z, c, \nu_{\alpha}^{2} ; \sigma_{\alpha}\right)=- & i \int_{0}^{\infty} d t \frac{e^{i z t} e^{-c t^{2} /(1-i t)}}{(1-i t)^{q}\left(1-i \sigma_{\alpha} t\right)^{p}} \\
& \times \mathcal{H}_{l}\left(\frac{\nu_{\alpha}^{2}}{1-i \sigma_{\alpha} t}\right) .
\end{aligned}
$$

The function $R_{q, p}^{(l)}$ is similar to that defined in Eq. (7) of Ref. [10]. It can be written in terms of the function $W_{q, p}$ previously analyzed by using expansion (A6) of Ref. [10]:

$$
\mathcal{H}_{n}(z)=\left(\frac{z}{2}\right)^{n} \sum_{k=0}^{\infty} \frac{(-)^{k}[2(n+k)] !}{(n+k) !(2 n+k) ! k !}\left(\frac{z}{2}\right)^{k} .
$$


As we have said previously, this expansion is only useful for computational purposes in the small Larmor radius approximation.

\section{E. Nonrelativistic approximation}

The nonrelativistic approximation for the effective dielectric tensor of a Maxwellian plasma can be obtained from Eq. (3) by the use of $\gamma \approx 1$ and by using the distribution (4). In this case, the tensor $\overleftrightarrow{\varepsilon}$ still is given by Eqs. (6), but with a slightly modified inhomogeneous PDF. Instead of expression (5), the nonrelativistic inhomogeneous PDF will be given by

$$
\begin{aligned}
\mathcal{G}_{r, p, m, l}= & -i \int_{0}^{\infty} d t \frac{(i t)^{r} e^{i z t}}{\left(1+i \zeta_{\alpha} t\right)^{p}} e^{-\beta t^{2}} e^{-\left(\nu_{\alpha}^{2}+\chi_{n \alpha}^{2} t^{2}\right) /\left(1+i \zeta_{\alpha} t\right)} \\
& \times \frac{\left[H_{n \alpha}(t)\right]^{m}}{\left[S_{n \alpha}(t)\right]^{l}} I_{l}\left(\frac{S_{n \alpha}(t)}{1+i \zeta_{\alpha} t}\right)
\end{aligned}
$$

where $\zeta_{\alpha}=1-\sigma_{\alpha}$. Equation (24) can be obtained from Eq. (5) by replacing 0 for $i t$ and $-\zeta_{\alpha}$ for $\sigma_{\alpha}$. In this case, of course, the inhomogeneous PDF $\mathcal{G}$ is no longer dependent on the quantity $q$.

Equation (24) is valid for arbitrary directions of propagation. However, the particular case of parallel propagation $(\psi=0)$ deserves special comments. In the case of parallel propagation, $\zeta_{\alpha}=0$, it is possible to use representation (12) and arrive at

$$
\begin{aligned}
\mathcal{G}_{r, p, m, l}= & \frac{-i}{(2 l-1) ! ! \pi} \int_{-1}^{1} d y\left(1-y^{2}\right)^{l-1 / 2} e^{-\nu_{\alpha}^{2}(1-y)} \\
& \times \int_{0}^{\infty} d t(i t)^{r} e^{i z t} e^{-\left[\beta+\chi_{n \alpha}^{2}(1+y)\right] t^{2}}\left(\nu_{\alpha}^{2}-\chi_{n \alpha}^{2} t^{2}\right)^{m} .
\end{aligned}
$$

Expanding now the last term in powers of $t$ and rearranging the time integral, we can easily express the nonrelativistic inhomogeneous PDF in terms of derivatives of the Fried and Conte function:

$$
\begin{aligned}
\mathcal{G}_{r, p, m, l}= & \frac{-\nu_{\alpha}^{2 m}}{(2 l-1) ! ! \pi} \sum_{j=0}^{m}\left(\begin{array}{c}
m \\
j
\end{array}\right) \frac{\left(\chi_{n \alpha} / \nu_{\alpha}\right)^{2 j}}{\left(2 \mu_{\alpha}^{1 / 2}\right)^{r+2 j+1}} \\
& \times \int_{-1}^{1} d y \frac{\left(1-y^{2}\right)^{l-1 / 2}}{\left|\gamma_{n}(y)\right|^{r+2 j+1}} e^{-\nu_{\alpha}^{2}(1-y)} \\
& \times Z^{(r+2 j)}\left(\frac{\mu_{\alpha}^{1 / 2} \delta_{n \alpha}}{2\left|\gamma_{n}(y)\right|}\right),
\end{aligned}
$$

where $\gamma_{n}^{2}(y) \equiv N_{\|}^{2} / 2+\rho_{n}^{2}(1+y)$, and

$$
\begin{gathered}
Z^{(n)}(z)=\frac{d^{n}}{d z^{n}} Z(z)=i 2^{n+1} e^{-z^{2}} \int_{-\infty}^{i z} d t e^{-t^{2}}(z+i t)^{n} \\
Z(z)=2 i e^{-z^{2}} \int_{-\infty}^{i z} d t e^{-t^{2}} .
\end{gathered}
$$

\section{APPLICATION FOR THE CASE OF ORDINARY MODE WAVES}

For the case of electromagnetic waves propagating perpendicularly to the magnetic field in a magnetized plasma, and velocity distributions which are even in $p_{\|}$, the dispersion relation factors into two branches, known as the ordinary and the extraordinary modes. Under these conditions the ordinary mode is specially suited to be used as an example for application of our formalism, since the dispersion relation depends on a single component of the dielectric tensor, and can be written as

$$
N_{\perp}^{2}=\varepsilon_{33}
$$

Using Eq. (6) and considering only one electron population, with loss-cone index $l_{\alpha}=l_{e}=1$ and equal perpendicular and parallel temperatures, the $\varepsilon_{33}$ component can be formally written in terms of the inhomogeneous dispersion function as

$$
\begin{aligned}
\varepsilon_{33}= & 1-X_{e}+\mu_{e} X_{e} \sum_{n=0, \pm 1}\left(\mathcal{G}_{0,3 / 2,1,|n|,|n|}\left(z, 0,1, \nu_{e}, \chi_{n e}, 1\right)\right. \\
& \left.+\left.\frac{\partial}{\partial h} \mathcal{G}_{0,3 / 2,1,|n|,|n|}^{*}\left(z, 0,1, \nu_{e}, \chi_{n e}, 1 ; h\right)\right|_{h=0}\right),
\end{aligned}
$$

where we have only considered harmonics $n=0, \pm 1$, since we are interested in wave frequencies near the electron cyclotron frequency, and where we have introduced $\mu_{e}=\mu_{\perp}=\mu_{\|}$.

In what follows, we only consider for simplicity the case of wave propagation parallel to the direction of inhomogeneity $(\psi=0)$. Evaluating the derivative of the dispersion function, as indicated, we arrive at the following explicit expression for the dispersion relation:

$$
\begin{gathered}
N_{\perp}^{2}=1-X_{e}+\mu_{e} X_{e}\left[\mathcal{G}_{0,3 / 2,1,0,0}-\mathcal{G}_{0,3 / 2,2,0,0}+\nu_{e}^{2} \mathcal{G}_{0,3 / 2,3,0,0}\right. \\
\left.-\mathcal{G}_{0,3 / 2,3,2,1}\right]+\mu_{e} X_{e} \sum_{n= \pm 1}\left[\mathcal{G}_{0,3 / 2,1,1,1}-2 \mathcal{G}_{0,3 / 2,2,1,1}\right. \\
\left.\quad+\nu_{e}^{2} \mathcal{G}_{0,3 / 2,3,1,1}-\mathcal{G}_{0,3 / 2,3,3,2}-\chi_{1 \alpha}^{2} \mathcal{G}_{2,3 / 2,3,1,1}\right]
\end{gathered}
$$

A simplified version of the dispersion relation (28) can be found with the use of the approximation given by Eq. (14), valid when the Larmor radius of the particles is small. It is also possible to assume that $\chi_{n e}^{2} \ll 1$, an assumption valid when $N_{B} \ll 2 / \sqrt{\mu_{e}}$, which is easily satisfied by weakly relativistic electrons near the cyclotron resonance. Moreover, for the harmonics $n=0,-1$, it is easy to show that the Shkarofsky functions can be approximated by

$$
\mathcal{F}_{q, r}(n=0) \approx \frac{1}{\mu_{e}}, \quad \mathcal{F}_{q, r}(n=-1) \approx \frac{1}{z-\beta} .
$$

The term corresponding to the $n=-1$ harmonic is negligible when compared to the $n=0$ term and can therefore be discarded.

Substituting Eq. (14) in Eq. (28) and neglecting all terms proportional to $\chi_{1 e}^{4}, \chi_{1 e}^{2} \nu_{e}^{2}, \chi_{1 e}^{4} \nu_{e}^{2}$, and $\mu_{e}^{-1}$, we arrive, after some algebra, at 


$$
\begin{gathered}
\varepsilon_{33}=1-X_{e}+N_{\perp}^{2} \zeta_{33}+N_{B}^{2} \psi_{33}, \\
\zeta_{33}=\frac{\mu_{e}}{\pi} \frac{X_{e}}{Y_{e}^{2}}\left[\int_{-1}^{1} d y\left(1-y^{2}\right)^{1 / 2} \mathcal{F}_{7 / 2}-\int_{-1}^{1} d y \frac{\mathcal{F}_{9 / 2}}{\left(1-y^{2}\right)^{1 / 2}}\right], \\
\psi_{33}=\frac{X_{e} \mu_{e}^{2}}{4 \pi}\left[\int_{-1}^{1} d y\left(1-y^{2}\right)^{1 / 2} \mathcal{F}_{7 / 2,2}-\int_{-1}^{1} d y \frac{\mathcal{F}_{9 / 2,2}}{\left(1-y^{2}\right)^{1 / 2}}\right],
\end{gathered}
$$

where $\mathcal{F}_{q, r}=\mathcal{F}_{q, r}\left(z, \chi_{1 e}^{2}(1+y)\right)$. The dispersion relation (26) is now simply written as

$$
N_{\perp}^{2}=\frac{1-X_{e}+N_{B}^{2} \psi_{33}}{1-\zeta_{33}} .
$$

The homogeneous limit can be readily found from Eq. (29). By making $N_{B} \rightarrow 0$, the integrals in Eq. (29) can be evaluated and $\varepsilon_{33}$ reduces to

$$
\begin{aligned}
\varepsilon_{33}= & 1-X_{e}+\frac{N_{\perp}^{2} X_{e}}{2 Y_{e}^{2}}\left\{F_{7 / 2}\left[\mu_{e}\left(1-\left|Y_{e}\right|\right)\right]\right. \\
& \left.-2 F_{9 / 2}\left[\mu_{e}\left(1-\left|Y_{e}\right|\right)\right]\right\},
\end{aligned}
$$

where $F_{q}(z)$ is the Dnestrovskii function [7]. This is the same expression obtained from the usual treatment of electron cyclotron waves propagating in a homogeneous magnetoplasma with a DGH distribution, using the small Larmor radius approximation [12].

The dispersion relations given by Eqs. (28) and (30) can now be solved, for a given set of parameters. Equation (28) utilizes the full inhomogeneous plasma dispersion function defined in the present manuscript, with the refraction index also appearing in the right-hand side. It is solved with the use of a straightforward numerical procedure which finds the desired $N_{\perp}$, for each value of $\omega, T_{e}, X_{e} / Y_{e}^{2}$, and $N_{B}$. The dispersion functions $\mathcal{G}$ are evaluated with the use of Eq. (13) in terms of Shkarofsky functions, using the relationship [7]

$$
\mathcal{F}_{q, r}(z, a)=\sum_{j=0}^{r}(-1)^{r}\left(\begin{array}{l}
r \\
j
\end{array}\right) \mathcal{F}_{q}(z, a) .
$$

The approximated dispersion relation given by Eq. (30), on the other hand, features the refraction index explicitly given by the right-hand side, and therefore there is no need of any root-solving procedure. In the right-hand side of the dispersion relation also appear Shkarofsky functions, which are also evaluated with the use of Eq. (31).

As an example of application of these dispersion relations, we consider $T_{e}=5 \mathrm{keV}, X_{e} / Y_{e}^{2}=0.5$, with the loss-cone index for the electronic distribution given by $l_{e}=1$. For these parameters, the results obtained from Eqs. (28) and (30) are virtually the same and cannot be easily distinguished in the graphics which follow. In Fig. 1 we show the real part of $N_{\perp}$ obtained from the dispersion relation, for several values of the inhomogeneity parameter $\widetilde{N}_{B}=c k_{B} /\left|\Omega_{e}\right|\left(\widetilde{N}_{B}=0,2 \times 10^{-3}, 4 \times 10^{-3}, 6 \times 10^{-3}\right.$, $8 \times 10^{-3}$, and $\left.1 \times 10^{-2}\right)$.

Figure 2 shows the imaginary part of $N_{\perp}$, for the same parameters. It is possible to observe the gradual displacement

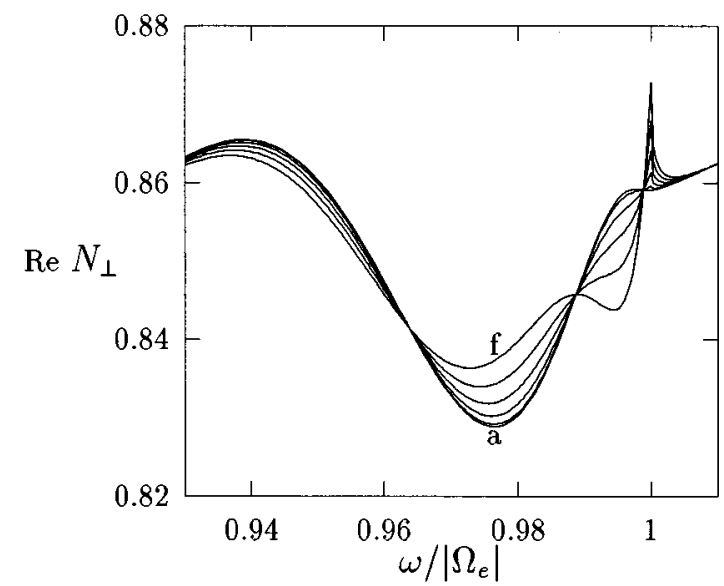

FIG. 1. Real part of the refraction index for ordinary mode waves vs normalized frequency, for several values of the inhomogeneity parameter $\widetilde{N}_{B} \quad\left(\widetilde{N}_{B}=0,2 \times 10^{-3}, 4 \times 10^{-3}, 6 \times 10^{-3}\right.$, $8 \times 10^{-3}$, and $\left.1 \times 10^{-2}\right)$. Curve $a, \widetilde{N}_{B}=0$; curve $f, \widetilde{N}_{B}=1 \times 10^{-2}$; the curves corresponding to the other values of $\widetilde{N}_{B}$ appear between the curves indicated by $a$ and $f$, for increasing $\widetilde{N}_{B}$. The parameters considered are $l_{e}=1, T_{\perp}=T_{\|}=T_{e}=5 \mathrm{keV}, X_{e} / Y_{e}^{2}=0.5, N_{\|}=0$, and $\psi=0$.

of the frequency of maximum amplification, which approaches the electron cyclotron frequency as the inhomogeneity is increased.

The effect of the inhomogeneity on the wave amplification may not appear substantial from the point of view of the local spectrum of emission of electron cyclotron waves, but it could be relevant in the case of a nonlocal analysis of wave amplification using, for instance, a ray-tracing routine coupled to the solution of the dispersion relation. Such an impressive task would be well beyond the scope of the present work, but it is nevertheless mentioned in order to

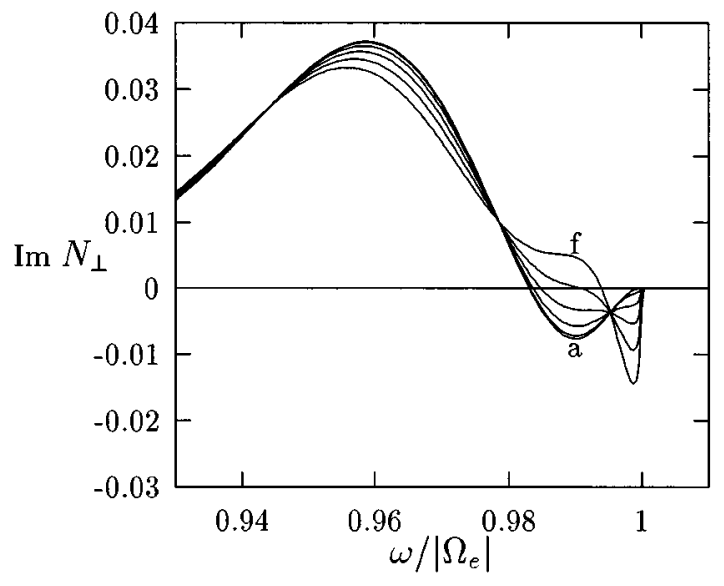

FIG. 2. Imaginary part of the refraction index for ordinary mode waves vs normalized frequency, for several values of the inhomogeneity parameter $\widetilde{N}_{B} \quad\left(\widetilde{N}_{B}=0,2 \times 10^{-3}, 4 \times 10^{-3}, 6 \times 10^{-3}\right.$, $8 \times 10^{-3}$, and $\left.1 \times 10^{-2}\right)$. Curve $a, \widetilde{N}_{B}=0$; curve $f, \widetilde{N}_{B}=1 \times 10^{-2}$; the curves corresponding to the other values of $\widetilde{N}_{B}$ appear between the curves indicated by $a$ and $f$, for increasing $\widetilde{N}_{B}$. The parameters considered are $l_{e}=1, T_{\perp}=T_{\|}=T_{e}=5 \mathrm{keV}, X_{e} / Y_{e}^{2}=0.5, N_{\|}=0$, and $\psi=0$. 


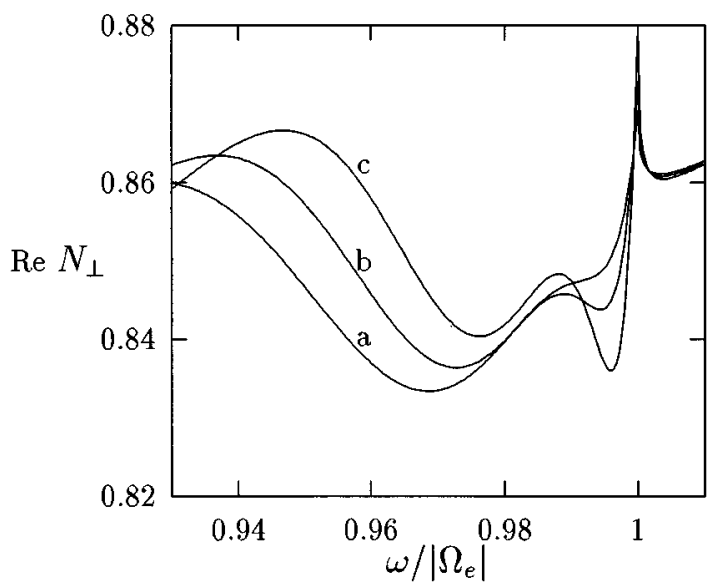

FIG. 3. Real part of the refraction index for ordinary mode waves vs normalized frequency, for curve $a, T_{e}=4 \mathrm{keV}$; curve $b$, $T_{e}=5 \mathrm{keV}$; and curve $c, T_{e}=6 \mathrm{keV}$. The parameters considered are $l_{e}=1, \quad X_{e} / Y_{e}^{2}=0.5, \quad N_{\|}=0, \quad \psi=0, \quad$ and $\quad \widetilde{N}_{B}=1 \times 10^{-2}, \quad$ for $T_{\perp}=T_{\|}=T_{e}$.

point out possible uses and extensions of the present formalism. For instance, inhomogeneity effects could play an important role in the study of the wave emission process, dubbed as auroral kilometric radiation, that occur on the auroral zone of earth's magnetosphere. Although the maser mechanism is the most probable candidate to explain this emission process, recent nonlocal analysis, using a locally homogeneous approximation for the dielectric tensor, obtained a total amplification that only marginally accounted for the observations $[13,14]$.

Another potential application was also mentioned in the literature, connected to a different range of frequencies. Recent publications have suggested that the explicit effect of the inhomogeneity should be taken into account in order to correctly reproduce the spectrum of ion-cyclotron waves observed on the plasma depletion layer, inside the sunward side of the magnetopause of earth's magnetosphere [15]. It is our intention to investigate the possibility of extension of the present formalism to the ion-cyclotron range of frequencies, in order to be able to study this phenomenon.

Other results obtained for the ordinary mode are shown in Figs. 3 and 4, in which we analyze the temperature dependence of the inhomogeneity effect for the ordinary mode, for $\widetilde{N}_{B}=1 \times 10^{-2}$ and three values of $T_{e}\left(T_{e}=4 \mathrm{keV}, T_{e}=5\right.$ $\mathrm{keV}$, and $T_{e}=6 \mathrm{keV}$ ), with the other parameters the same as in Figs. 1 and 2.

It can be easily seen that only a small variation on the temperature has a large effect on the instability. The lower the temperature, the greater the maximum amplification and the narrower the spectrum of emission. This behavior can be qualitatively understood by the analysis of the resonance condition of the dielectric tensor associated to a plasma with an inhomogeneous magnetic field. The inhomogeneous terms of the resonance condition become important for wave emission (or absorption) when propagation nearly perpendicular to the ambient magnetic field is considered and when the temperature is decreased. This analysis is made in Appendix C.

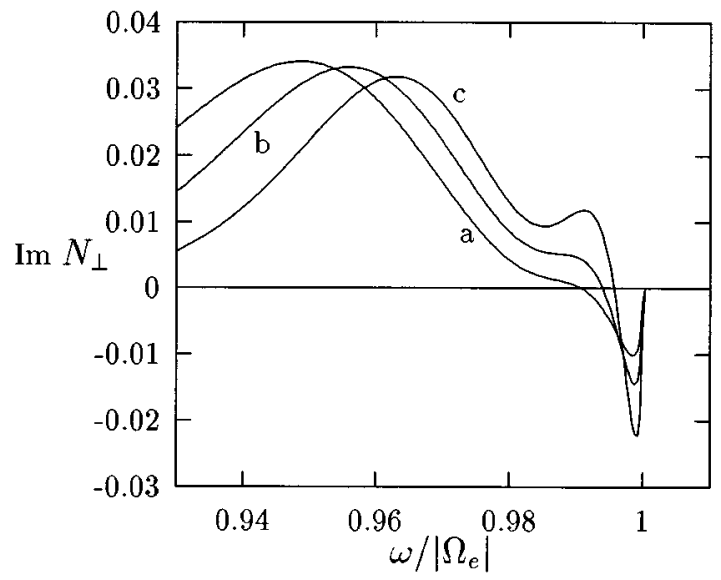

FIG. 4. Imaginary part of the refraction index for ordinary mode waves vs normalized frequency, for curve $a, T_{e}=4 \mathrm{keV}$; curve $b$, $T_{e}=5 \mathrm{keV}$; and curve $c, T_{e}=6 \mathrm{keV}$. The parameters considered are $l_{e}=1, \quad X_{e} / Y_{e}^{2}=0.5, \quad N_{\|}=0, \quad \psi=0, \quad$ and $\quad \widetilde{N}_{B}=1 \times 10^{-2}, \quad$ for $T_{\perp}=T_{\|}=T_{e}$.

\section{CONCLUSIONS}

In the present paper we have resumed the study of the dielectric properties of plasmas in a weakly inhomogeneous magnetic field, using recently derived expressions for the components of an effective dielectric tensor to be utilized in the dispersion relation, which have been derived according to the BGI procedure [5]. We have rewritten these components for the case of a loss-cone distribution function, using the weakly relativistic approximation and introducing the socalled "inhomogeneous plasma dispersion function," which has been shown to be related to other well-known plasma dispersion functions appearing in the case of homogeneous media. The relationship between the inhomogeneous plasma dispersion function and other dispersion functions such as the Shkarofsky and Dnestrovskii functions (and even the $Z$ function, in the nonrelativistic case) is important not only for computational purposes, due to the well studied analytical properties of these functions and due to the existence of sum rules and other useful mathematical relationships, but also because it enabled us to obtain very important analytical properties of the $\mathcal{G}$ function. For instance, we have shown that the anti-Hermitian part of the dielectric tensor is entirely due to the imaginary part of the $\mathcal{G}$ function, which is nonvanishing only in the case of wave-particle resonance. The limit cases of propagation parallel, perpendicular, and oblique to the inhomogeneity direction have been considered, as well as the weakly relativistic and nonrelativistic cases. As an application, we have considered the case of ordinary mode waves propagating perpendicularly to the magnetic field, along the direction of the inhomogeneity, demonstrating the displacement of the frequency of maximum amplification which is a consequence of the inhomogeneity. It is worthwhile to point out that other formulations appearing in the literature, which do not include the frequency correction due to the inhomogeneity, do not predict any effect due to the inhomogeneity for propagation parallel to the direction of the inhomogeneity [16].

We believe that the displacement of the frequency of maximum amplification due to the inhomogeneity, coupled 
to the modification of the real part of the refraction index, can have important consequences on the total amplification of the wave as it propagates inside a nonuniform medium, a situation which therefore deserves to be investigated. The importance of taking explicitly into account the effect of the inhomogeneity on the amplification of waves has also been recently stressed by other authors, working on emission processes that occur inside the magnetopause [15].

It is our intention to apply the general expressions obtained for the inhomogeneous plasma dispersion function for further studies of wave propagation in weakly relativistic plasmas with inhomogeneous magnetic fields. It may appear that for useful applications the inhomogeneous plasma dispersion function as given by Eqs. (13), (17), and (22) may be difficult to handle, due to the number of integrations and summations over different variables and indexes. These expressions are quite general and no attempt has been made to simplify them beyond the intrinsic limitations of its derivation, detailed in Ref. [5] and listed in Sec. II of the present paper, because the effective dielectric tensor written in terms of the inhomogeneous dispersion function features important properties, which do not depend on any particular approximation. We have seen in Sec. V an application to the case of ordinary mode waves, which illustrate the practical use of the formulation. This application is quite simple, but it is possible to demonstrate that, for many cases of practical interest, the general expressions are quite easy to handle in a numerical application, due to the fast convergence of the series involved, and only a small number of terms has actually to be considered in the calculation. We may be more explicit about the convergence of these expansions by considering Eq. (22), which is the expression featuring the greatest complexity, since it has two power series expansions on the quantity $\left(1-\sigma_{\alpha}^{-1}\right)$ [see definitions after Eq. (22) and the expansion given by Eq. (18)] and other two power series on the quantity $\nu_{\alpha}^{2}$ [one explicit in Eq. (22) and the other in the expansion of $\mathcal{H}_{n}$, Eq. (23)]. However, each pair of series expansions can easily be rearranged in order to become one series and one finite summation, therefore reducing the number of expansions to only one in Eq. (17) and two in Eq. (22), with the magnitude of $\nu_{\alpha}^{2}$ and $\left(1-\sigma_{\alpha}^{-1}\right)$ being important in the determination of the convergence velocity of these series. The quantity $\nu_{\alpha}^{2}$ is nothing but the usual small Larmor radius expansion parameter and can be handled as in the related homogeneous treatment. The other quantity,

$$
1-\sigma_{\alpha}^{-1}=\frac{-\widetilde{N}_{B} N_{\perp} \sin \psi}{1-\widetilde{N}_{B} N_{\perp} \sin \psi}, \quad \widetilde{N}_{B}=\frac{\epsilon c}{\Omega_{\alpha}}=\frac{N_{B}}{Y_{\alpha}}
$$

is related to the inhomogeneity and can be even smaller than $\nu_{\alpha}^{2}$ for typical situations. Moreover, in many cases of practical applications, only one electron population is present, which eliminates the summation over species in the dielectric tensor. Also the summation over harmonics can usually be disregarded, since in weakly relativistic situations, for frequencies near the electron cyclotron frequency, only one harmonic term is relevant for absorption studies.

The conclusion is that the apparently cumbersome expressions for the inhomogeneous dispersion relation can be handled for numerical applications similarly to the well- known plasma dispersion functions used for the case of homogeneous plasmas. We have seen an example of the approximations which can be made, in the derivation of the approximated dispersion relation (30), with nearly the same numerical results as those obtained from the complete expression given by Eq. (28).

It is also worthwhile to point out in these conclusions that along the derivation we have mentioned that the effective dielectric tensor satisfies Onsager symmetry relations, as expected from the time invariance from the microscopic equations of motion. In contrast, other approaches of dielectric description of inhomogeneous media which do not incorporate the BGI corrections do not satisfy Onsager symmetry. While discussing the proper symmetry properties of the dielectric tensor, it can be explicitly shown that the antiHermitian parts of the effective dielectric tensor are only connected to resonant terms, which should be expected within the framework of the linear kinetic theory, since in this case the only energy transfer process is related to the wave-particle resonance. However, as has been discussed in a previous publication [5], alternative expressions for the dielectric tensor of an inhomogeneous plasma that do not obey Onsager symmetry show contributions from nonresonant particles in the anti-Hermitian part. Because the effective dielectric tensor obeys Onsager symmetry relations, its antiHermitian part naturally contains contributions only from resonant particles. We have incorporated this demonstration in Appendix B. As a consequence of this property, the imaginary part of the refraction index obtained from the dispersion relation, when the effective dielectric tensor is utilized, really describes wave-particle energy exchange due to wave dissipation or amplification.

The importance of satisfying Onsager relations was also pointed out in a recent study of the onset of lower-hybriddrift instability in the magnetotail of Earth's magnetosphere, in which it is recognized that the usual approach to study the effect of cross-field instabilities on particle acceleration leads to nonsymmetric components of the dielectric tensor [17].

With the BGI formalism, we have shown that even in very different circumstances, like inhomogeneities in density and temperature and gradients of the magnetic field, the dielectric tensor is naturally derived with the correct symmetry properties. Therefore it is the correct quantity to be used in the dispersion relation that describes wave emission and propagation in an inhomogeneous plasma.

In Appendix $\mathrm{C}$, some limit cases of the wave-particle resonance condition in inhomogeneous media are discussed.

\section{ACKNOWLEDGMENTS}

This work has been partially supported by the Brazilian agencies Conselho Nacional de Desenvolvimento Científico e Tecnológico (CNPq), Fundacão de Amparo à Pesquisa do Estado do Rio Grande do Sul (FAPERGS), and Financiadora de Estudos e Projetos (FINEP).

\section{APPENDIX A: THE MOMENTUM INTEGRALS AND THE DEFINITION OF THE INHOMOGENEOUS PDF}

In the components of the effective dielectric tensor we have the momentum integrals 


$$
\int d^{3} u u_{\perp} \mathcal{L}\left(f_{\alpha 0}\right) e^{i D_{n \alpha} \tau}\left[F_{n \alpha}(\tau)\right]^{(|n|-1)} \frac{\Pi_{n \alpha}^{-} \Pi_{n \alpha}^{+}}{\left(W_{n}^{-} W_{n}^{+}\right)^{|n|}},
$$

where the components of the tensor $\boldsymbol{\Pi}_{n \alpha}^{ \pm}$are given in Eq. (3), as well as the definition of the quantity $F_{n \alpha}$.

Using the definition of $D_{n \alpha}$ from Eq. (1), and considering the weakly relativistic case, $\gamma \approx 1+u^{2} / 2$, this integral can be written as

$$
\begin{aligned}
\int & d^{3} u u_{\perp} \mathcal{L}\left(f_{\alpha 0}\right)\left[F_{n \alpha}(t)\right]^{(|n|-1)} \\
& \times \frac{\boldsymbol{\Pi}_{n \alpha}^{-} \Pi_{n \alpha}^{+}}{\left(W_{n}^{-} W_{n}^{+}\right)^{|n|}} e^{i \mu_{\alpha} \delta_{n \alpha} t-i N_{\|} \mu_{\alpha} u\left\|t+i \mu_{\alpha} u_{\perp}^{2} \sigma_{\alpha} t / 2+i \mu_{\alpha} u_{\|}^{2}\right\|^{t / 2}},
\end{aligned}
$$

where the dimensionless variable $t \equiv(\omega / \mu) \tau$ has been introduced. The quantities $\delta_{n \alpha}$ and $\sigma_{\alpha}$ are defined in Eq. (5).

By considering the case of an isotropic Maxwellian distribution, given by Eq. (4) for $l_{\alpha}=0$ and equal parallel and perpendicular temperatures, we arrive at

$$
\begin{aligned}
& -n_{\alpha} \mu_{\alpha}\left(\frac{\mu_{\alpha}}{2 \pi}\right)^{3 / 2} \int d^{3} u u_{\perp}^{2}\left[F_{n \alpha}(t)\right]^{(|n|-1)} \frac{\boldsymbol{\Pi}_{n \alpha}^{-} \boldsymbol{\Pi}_{n \alpha}^{+}}{\left(W_{n}^{-} W_{n}^{+}\right)^{|n|}}
\end{aligned}
$$

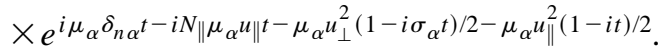

Considering that the quantities $W_{n}^{ \pm}$and $F_{n \alpha}$ are not depending on the parallel momentum, and considering that the quantity $u_{\|}$only appears linearly in the components of the tensor $\Pi_{n \alpha}^{ \pm}$, it is seen that the parallel integrals which will appear in the calculation will be

$$
I_{\|}^{j}=\int_{-\infty}^{\infty} d u_{\|} e^{-\mu_{\alpha} u_{\|}^{2}(1-i t) / 2-i N_{\|} \mu_{\alpha} u_{\|} u_{\|}^{j},}
$$

with $j$ ranging from 0 to 2 .

These parallel integrals are easily performed, with the result

$$
I_{\|}^{\dot{H}}=\left(\frac{2 \pi}{\mu_{\alpha}}\right)^{1 / 2} \frac{e^{-\beta t^{2} /(1-i t)}}{(1-i t)^{1 / 2}} J_{\|}^{\dot{1}},
$$

where

$$
J_{\|}^{j}=\sum_{r=0}^{j}\left(\begin{array}{l}
j \\
r
\end{array}\right) \frac{\left[1+(-1)^{r}\right]}{2 \mu_{\alpha}^{r / 2}}(r-1) ! !\left(\frac{-i N_{\|} t}{1-i t}\right)^{j-r}(1-i t)^{-r / 2},
$$

and where we defined $(-1) ! !=1$.

After explicit use of the components of the tensor $\boldsymbol{\Pi}_{n \alpha}^{ \pm}$ and of the quantities $W_{n}^{ \pm}$and $F_{n \alpha}$, it is seen that the $u_{\perp}$ integrals which are useful for the calculation are

$$
\begin{gathered}
I_{\perp 1}=\int_{0}^{\infty} d u_{\perp} u_{\perp} e^{-\mu_{\alpha}\left(1-i \sigma_{\alpha} t\right) u_{\perp}^{2} / 2} J_{|n|}\left(W_{n}^{+}\right) J_{|n|}\left(W_{n}^{-}\right), \\
I_{\perp 2}^{ \pm}=\int_{0}^{\infty} d u_{\perp} u_{\perp}^{2} e^{-\mu_{\alpha}\left(1-i \sigma_{\alpha} t\right) u_{\perp}^{2} / 2} J_{|n|}\left(W_{n}^{\mp}\right) J_{|n|+1}\left(W_{n}^{ \pm}\right), \\
I_{\perp 3}=\int_{0}^{\infty} d u_{\perp} u_{\perp}^{3} e^{-\mu_{\alpha}\left(1-i \sigma_{\alpha} t\right) u_{\perp}^{2} / 2} J_{|n|+1}\left(W_{n}^{+}\right) J_{|n|+1}\left(W_{n}^{-}\right) .
\end{gathered}
$$

These integrals can be easily solved, and are given by simple expressions involving the modified Bessel function $I_{n}[9]$

$$
\begin{gathered}
I_{\perp 1}=\frac{e^{-\left(\nu_{\alpha}^{2}+\chi_{n \alpha}^{2} t^{2}\right) /\left(1-i \sigma_{\alpha} t\right)}}{\mu_{\alpha}\left(1-i \sigma_{\alpha} t\right)} I_{|n|}\left(\frac{S_{n \alpha}(t)}{1-i \sigma_{\alpha} t}\right), \\
I_{\perp 2}^{ \pm}=\frac{e^{-\left(\nu_{\alpha}^{2}+\chi_{n \alpha}^{2} t^{2}\right) /\left(1-i \sigma_{\alpha} t\right)}}{\mu_{\alpha}^{3 / 2}\left(1-i \sigma_{\alpha} t\right)^{2}}\left[T_{n}^{ \pm} I_{|n|}\left(\frac{S_{n \alpha}(t)}{1-i \sigma_{\alpha} t}\right)\right. \\
\left.-T_{n}^{\mp} I_{|n|+1}\left(\frac{S_{n \alpha}(t)}{1-i \sigma_{\alpha} t}\right)\right], \\
I_{\perp 3}=2 \frac{e^{-\left(\nu_{\alpha}^{2}+\chi_{n \alpha}^{2} t^{2}\right) /\left(1-i \sigma_{\alpha} t\right)}}{\mu_{\alpha}^{2}\left(1-i \sigma_{\alpha} t\right)^{2}}\left[\frac{S_{n \alpha}(t)}{1-i \sigma_{\alpha} t} I_{|n|}\left(\frac{S_{n \alpha}(t)}{1-i \sigma_{\alpha} t}\right)\right. \\
\left.-\left(|n|+\frac{\nu_{\alpha}^{2}+\chi_{n \alpha}^{2} t^{2}}{1-i \sigma_{\alpha} t}\right) I_{|n|+1}\left(\frac{S_{n \alpha}(t)}{1-i \sigma_{\alpha} t}\right)\right],
\end{gathered}
$$

where $T_{n}^{ \pm}=\sqrt{\nu_{\alpha}^{2} \pm 2 \nu_{\alpha} \cos (\psi) \chi_{n \alpha} t+\chi_{n \alpha}^{2} t^{2}}$.

In these integrals given by Eqs. (A5) and (A7) it is possible to see the basic elements appearing in the definition of the inhomogeneous plasma dispersion function appearing in Eq. (5). It is therefore a straightforward and tedious task to use these integrals and express the components of the effective dielectric tensor for loss-cone distributions with $l_{\alpha} \neq 0$ and $r_{T} \neq 1$ in terms of the inhomogeneous PDF, as given by Eqs. (6).

\section{APPENDIX B: THE ANTI-HERMITIAN PART OF THE EFFECTIVE DIELECTRIC TENSOR}

General properties featured by the effective dielectric tensor can be inferred either from the integral expression (1) or from the formulation in terms of the inhomogeneous plasma dispersion function, as well as from the limit cases. For instance, it has been shown that the BGI transformation applied to a plane wave approximation for the dielectric tensor components restores an important property which should be expected for the dielectric tensor, from a physical point of view [5]. This property is the Onsager symmetry, which can be confirmed by direct inspection of Eqs. (1) or (3). There is another property, which is a consequence of the former and which is of paramount importance in the study of absorption or emission of radiation by inhomogeneous magnetoplasmas. Namely, the fact that the anti-Hermitian part of $\overleftrightarrow{\varepsilon}$ must be only due to resonant particles, in order that the absorption coefficient obtained from the dispersion relation really describes wave energy dissipation (or amplification).

It is easy to show that the anti-Hermitian part of the tensor (6) satisfies this condition. The anti-Hermitian part of a tensor $\overleftrightarrow{\varepsilon}$ is defined as

$$
\varepsilon_{i j}^{\mathrm{aH}}=\frac{\varepsilon_{i j}-\varepsilon_{j i}^{*}}{2 i} .
$$

In what follows, we demonstrate only the case of the $\varepsilon_{12}$ component. The case of the other components is similar and features less complexity. We also particularize for the case $l_{\alpha}=0$ and $r_{T}=1$, for simplicity. Calculating the antiHermitian part of $\varepsilon_{12}$ from Eq. (6), according to definition (B1), we obtain 


$$
\begin{aligned}
\varepsilon_{12}^{\mathrm{aH}}= & -\sum_{\alpha} \mu_{\alpha} X_{\alpha} \sum_{n \rightarrow-\infty}^{\infty}\left\{i 2 \nu_{\alpha} \sin (\psi) \chi_{n \alpha}\left[\mathcal{G}_{1,1 / 2,3,|n|,|n|}^{\prime \prime}-\nu_{\alpha}^{2} \mathcal{G}_{1,1 / 2,3,|n|,|n|+1}^{\prime \prime}+\chi_{n \alpha}^{2} \mathcal{G}_{3,1 / 2,3,|n|,|n|+1}^{\prime \prime}\right]\right. \\
& +\operatorname{in}\left\{|n| \mathcal{G}_{0,1 / 2,1,|n|-1,|n|}^{\prime \prime}-\nu_{\alpha}^{2}\left[\mathcal{G}_{0,1 / 2,2,|n|-1,|n|}^{\prime \prime}-\mathcal{G}_{0,1 / 2,2,|n|,|n|+1}^{\prime \prime}\right]+\chi_{n \alpha}^{2}\left[\mathcal{G}_{2,1 / 2,2,|n|-1,|n|}^{\prime \prime}+\mathcal{G}_{2,1 / 2,2,|n|,|n|+1}^{\prime \prime}\right]\right\}-\nu_{\alpha}^{2} \sin (2 \psi) \\
& \times\left[\mathcal{G}_{0,1 / 2,3,|n|,|n|}^{\prime \prime}-|n| \mathcal{G}_{0,1 / 2,2,|n|-1,|n|}^{\prime \prime}-\nu_{\alpha}^{2} \mathcal{G}_{0,1 / 2,3,|n|,|n|+1}^{\prime \prime}+\chi_{n \alpha}^{2} \mathcal{G}_{2,1 / 2,3,|n|,|n|+1}^{\prime \prime}\right]-2 n \nu_{\alpha} \cos (\psi) \chi_{n \alpha} \mathcal{G}_{1,1 / 2,2,|n|-1,|n|}^{\prime \prime},
\end{aligned}
$$

where $\mathcal{G}=\mathcal{G}^{\prime}+i \mathcal{G}^{\prime \prime}$. Therefore it is clearly shown that the anti-Hermitian part of $\varepsilon_{12}$ is entirely due to the imaginary part of the inhomogeneous PDF, which is nonvanishing only in the case of wave-particle resonance.

The connection between the imaginary part of the inhomogeneous PDF and the resonance condition can be demonstrated by looking at Eqs. (13), (19), and (22) for the weakly relativistic approximation, and at Eq. (25) for the nonrelativistic case. In the weakly relativistic case, all the expressions obtained show that the imaginary part of $\mathcal{G}$ is determined by the imaginary part of the generalized Shkarofsky function $\mathcal{F}_{q, r}(z, a)$, which is related to the Shkarofsky function $\mathcal{F}_{q}(z, a)$, as seen in Eq. (31).

The imaginary part of the $\mathcal{F}_{q}(z, a)$ function is given by

$$
\operatorname{Im}\left[\mathcal{F}_{q}(z, a)\right]= \begin{cases}0, & z-a \geqslant 0 \\ -\pi e^{z-2 a}[(a-z) / a]^{(q-1) / 2} I_{q-1}\left[2 a^{1 / 2}(a-z)^{1 / 2}\right], & z-a<0\end{cases}
$$

provided that $z$ and $a$ are real. $I_{q}(z)$ is the modified Bessel function of the first kind of fractional order.

According to our results, the factor $z-a$ appearing in the function $\mathcal{F}$ is given by

$$
z-a=\mu_{\alpha}\left(1-n Y_{\alpha}(x)-\frac{N_{\|}^{2}}{2}-\frac{n^{2} N_{B}^{2}}{4 \sigma_{\alpha}}(1+y)\right) .
$$

For a given choice of wave vector, wave frequency, and $\epsilon$, the function $\mathcal{F}$ is integrated in the variable $y$ ranging from -1 to 1 . The maximum contribution of the inhomogeneous term comes from $y \simeq 1$. Therefore a necessary condition for the existence of a nonvanishing imaginary part in the function $\mathcal{G}$ is

$$
1-n Y_{\alpha}(x)-\frac{N_{\|}^{2}}{2}-\frac{n^{2} N_{B}^{2}}{2 \sigma_{\alpha}}<0 .
$$

On the other hand, the resonance condition for the weakly relativistic case is given by Eq. (C6), appearing in Appendix C. We verify that the condition for existence of the imaginary part of $\mathcal{G}$ is exactly the condition for the existence of a resonance ellipse in momentum space.

In the nonrelativistic case, we have seen that the $\mathcal{G}$ function is given by Eq. (25), which is expressed in terms of the $Z$ function. The argument of the $Z$ function is real and is given by

$$
\mu_{\alpha}^{1 / 2} \frac{\delta_{n \alpha}}{\sqrt{2}\left|\gamma_{n}(y)\right|}=\mu_{\alpha}^{1 / 2} \frac{\delta_{n \alpha}}{\sqrt{N_{\|}^{2}+n^{2} N_{B}^{2}(1+y) / 2}} .
$$

Also in this case the maximum contribution for the inhomogeneous term comes from $y \simeq 1$, resulting in the argument of the $Z$ function becoming exactly proportional to the resonant velocity, as given by the right-hand side of the resonance condition for the non-relativistic approximation (C8).

Similar analysis applied to the tensor obtained with the plane wave approximation, without the use of the BGI correction, would result in anti-Hermitian parts featuring nonresonant contributions [5]. Therefore it is shown that the BGI correction also restores the proper contribution to the antiHermitian part of the dielectric tensor.

\section{APPENDIX C: THE RESONANCE CONDITION}

The complete resonance condition is given by the denominator in Eq. (12) of Ref. [5], and is repeated here for the sake of completeness:

$$
\begin{aligned}
\mathcal{D}_{n \alpha}= & \gamma \omega-k_{\|} c u_{\|}-n \Omega_{\alpha}(1+\epsilon x)-n \epsilon c u_{\perp} \sin \varphi \\
& -\epsilon \frac{k_{\perp} u_{\perp}^{2} c^{2}}{2 \Omega_{\alpha}} \sin \psi=0
\end{aligned}
$$

or

$$
\gamma-N_{\|} u_{\|}-n Y_{\alpha}(x)-n N_{B} u_{\perp} \sin \varphi-N_{B} \frac{N_{\perp} u_{\perp}^{2}}{2 Y_{\alpha}} \sin \psi=0 .
$$

The first three terms in Eq. (C1) come from the resonance condition of a homogeneous plasma. The last two terms are due to the inhomogeneity correction. It is clearly seen that inhomogeneity effects appear even when $\psi=0$, when the drift instability is absent, and may be significant near the cyclotron frequency of the $\alpha$ population. The inhomogeneity creates, in addition to the resonance broadenings due to Doppler and relativistic effects, a broadening due to the inhomogeneity. This may be important when the homogeneous contribution is small, even if the parameter $\epsilon$ is considered small.

This statement can be made clearer by considering the angular wave frequency which resonates with a particle with velocity $\boldsymbol{v}$, near the cyclotron frequency. From Eq. (C1), we have

$$
\omega=k_{\|} v_{\|}+n \frac{\Omega_{\alpha}}{\gamma}+n \epsilon v_{\perp} \sin \varphi+\epsilon \frac{k_{\perp} v_{\perp}^{2} \gamma}{2 \Omega_{\alpha}} \sin \psi
$$


If the particles have a velocity spread given by $v_{T}^{2} \propto T_{\alpha}$, then the first term in Eq. (C2) introduces an emission line broadening around the $n$th harmonic due to the Doppler effect given by $[18]$

$$
\left(\Delta \omega_{n}\right)_{1} \approx n \Omega_{\alpha}\left|N_{\|}\right|\left(\frac{v_{T}}{c}\right) .
$$

The second term corresponds to the "relativistic broadening" given by

$$
\left(\Delta \omega_{n}\right)_{2} \approx n \Omega_{\alpha}\left(\frac{v_{T}}{c}\right)^{2} .
$$

The last two terms correspond to "inhomogeneous broadenings,', which are given by

$$
\begin{gathered}
\left(\Delta \omega_{n}\right)_{3} \approx n^{2} \Omega_{\alpha} N_{B}\left(\frac{v_{T}}{c}\right), \\
\left(\Delta \omega_{n}\right)_{4} \approx n^{2} \Omega_{\alpha} N_{B}\left|N_{\perp}\right| \sin \psi\left(\frac{v_{T}}{c}\right)^{2} .
\end{gathered}
$$

Therefore the first inhomogeneous linewidth compared to the two homogeneous ones gives:

$$
\frac{\left(\Delta \omega_{n}\right)_{3}}{\left(\Delta \omega_{n}\right)_{1}}=\frac{n N_{B}}{N_{\|}}, \quad \frac{\left(\Delta \omega_{n}\right)_{3}}{\left(\Delta \omega_{n}\right)_{2}}=\frac{n N_{B}}{\left(v_{T} / c\right)},
$$

which shows that $\left(\Delta \omega_{n}\right)_{3}$ becomes important both when the wave vector approaches the perpendicular and when the temperature is decreased. At the same time, the other inhomogeneous broadening effect compared to the Doppler homogeneous contributions gives

$$
\frac{\left(\Delta \omega_{n}\right)_{4}}{\left(\Delta \omega_{n}\right)_{1}}=n N_{B}\left|\frac{N_{\perp}}{N_{\|}}\right|\left(\frac{v_{T}}{c}\right) \sin (\psi) .
$$

It is seen that, compared to the Doppler term, this inhomogeneous contribution becomes important when $N_{\|} \rightarrow 0$. However, contrasting with the case of term $\left(\Delta \omega_{n}\right)_{3}$, it also becomes important when the particle temperature is increased. Compared to the relativistic case, however, the contribution of the term $\left(\Delta \omega_{n}\right)_{4}$ is always small, except near resonances

$$
\frac{\left(\Delta \omega_{n}\right)_{4}}{\left(\Delta \omega_{n}\right)_{2}}=n N_{B}\left|N_{\perp}\right| \sin \psi
$$

In the next subsections we will consider some special cases of Eq. (C1).

\section{Waves with $\mathbf{k}_{\perp}$ parallel to the direction of the inhomogeneity $(\psi=0)$}

In this case the resonance condition reduces to

$$
\gamma-N_{\|} u_{\|}-n Y_{\alpha}(x)-n N_{B} u_{\perp} \sin \varphi=0,
$$

which can be decomposed in Cartesian coordinates to give the equation of a complicated surface in three dimensions:

$$
\begin{aligned}
u_{x}^{2}+ & \left(1-n^{2} N_{B}^{2}\right)\left(u_{y}-\frac{n^{2} N_{B} Y_{\alpha}(x)}{1-n^{2} N_{B}^{2}}\right)^{2} \\
& +\left(1-N_{\|}^{2}\right)\left(u_{\|}-\frac{n N_{\|} Y_{\alpha}(x)}{1-N_{\|}^{2}}\right)^{2}-2 n N_{B} N_{\|} u_{y} u_{\|} \\
= & n^{2} Y_{\alpha}^{2}(x)-1+\frac{n^{4} N_{B}^{2} Y_{\alpha}^{2}(x)}{1-n^{2} N_{B}^{2}}+\frac{n^{2} N_{\|}^{2} Y_{\alpha}^{2}(x)}{1-N_{\|}^{2}} .
\end{aligned}
$$

This complicated surface reduces to an ellipsoid of revolution when $N_{\|}=0$ :

$$
\begin{aligned}
u_{x}^{2}+ & \left(1-n^{2} N_{B}^{2}\right)\left(u_{y}-\frac{n^{2} N_{B} Y_{\alpha}(x)}{1-n^{2} N_{B}^{2}}\right)^{2}+u_{\|}^{2} \\
& =\frac{n^{2}\left[Y_{\alpha}^{2}(x)+N_{B}^{2}\right]-1}{1-n^{2} N_{B}^{2}} .
\end{aligned}
$$

In the homogeneous case, $N_{B}=0$, and the ellipsoid further reduces to the well-known "resonance circle" $u_{1}^{2}+u_{2}^{2}+u_{\|}^{2}=n^{2} Y_{\alpha}^{2}(x)-1$.

\section{Weakly relativistic approximation}

In this case we have $\gamma \approx 1+u^{2} / 2$, and the resonance condition (C1) also reduces to the equation of an ellipsoid

$$
\begin{gathered}
\frac{\sigma_{\alpha}}{2} u_{x}^{2}+\frac{\sigma_{\alpha}}{2}\left(u_{y}-\frac{n N_{B}}{\sigma_{\alpha}}\right)^{2}+\frac{1}{2}\left(u_{\|}-N_{\|}\right)^{2} \\
=n Y_{\alpha}(x)-1+\frac{N_{\|}^{2}}{2}+\frac{n^{2} N_{B}^{2}}{2 \sigma_{\alpha}} .
\end{gathered}
$$

Evidently, this ellipsoid exists if the right-hand side is positive. This is exactly the condition for the existence of the imaginary part of the $\mathcal{F}_{q}$ function, as given by Eq. (B2). It is clearly seen that the anti-Hermitian parts of the effective dielectric tensor are nonvanishing only in the case of waveparticle resonance.

\section{Nonrelativistic approximation}

In the nonrelativistic approximation the resonance condition can be written as

$$
n N_{B} u_{y}+N_{\|} u_{\|}=1-n Y_{\alpha}(x)
$$

which can be reduced to a simpler form by rotation of axes by an angle $\theta=-\tan ^{-1}\left(n N_{B} / N_{\|}\right)$:

$$
\overline{u_{\|}}=\frac{1-n Y_{\alpha}(x)}{\sqrt{n^{2} N_{B}^{2}+N_{\|}^{2}}} .
$$

The right-hand side of Eqs. (C6) and (C8) is related to the imaginary part of the inhomogeneous plasma dispersion function (5) and (25), as we show in Appendix B. 
[1] V. S. Beskin, A. V. Gurevich, and Y. I. Istomin, Zh. Éksp. Teor. Fiz. 92, 1277 (1987) [Sov. Phys. JETP 65, 715 (1987)].

[2] R. A. Caldela Filho, R. S. Schneider, and L. F. Ziebell, J. Plasma Phys. 42, 165 (1989).

[3] R. A. Caldela Filho, R. S. Schneider, and L. F. Ziebell, J. Plasma Phys. 43, 335 (1990).

[4] C. J. H. Cavalcanti, R. S. Schneider, and L. F. Ziebell, J. Plasma Phys. 52, 195 (1994).

[5] R. Gaelzer, R. S. Schneider, and L. F. Ziebell, Phys. Rev. E 51, 2407 (1995).

[6] R. A. Dory, G. E. Guest, and E. G. Harris, Phys. Rev. Lett. 14, 131 (1965).

[7] P. A. Robinson, J. Math. Phys. 27, 1206 (1986).

[8] P. A. Robinson, J. Math. Phys. 28, 1203 (1987).

[9] Handbook of Mathematical Functions, edited by $\mathrm{M}$. Abramowitz and I. A. Stegun (Dover Publications, New York, 1970).
[10] M. Bornatici, G. Chiozzi, and P. de Chiara, J. Plasma Phys. 44, 319 (1990)

[11] N. M. Temme, A. E. Sumner, and S. S. Sazhin, Astrophys. Space Sci. 194, 173 (1992).

[12] H. K. Wong et al., J. Plasma Phys. 28, 503 (1982).

[13] R. Gaelzer, L. F. Ziebell, and R. S. Schneider, J. Geophys. Res. 99, 8905 (1994).

[14] C. J. H. Cavalcanti, R. S. Schneider, and L. F. Ziebell, J. Geophys. Res. (1996).

[15] R. E. Denton, M. K. Hudson, S. A. Fuselier, and B. J. Anderson, J. Geophys. Res. 98, 13477 (1993).

[16] A. B. Mikhailovskii and O. G. Onishchenko, J. Plasma Phys. 37, 15 (1987).

[17] P. H. Yoon, A. T. Y. Lui, and C.-L. Chang, Phys. Plasmas 1, 3033 (1994).

[18] M. Bornatici, R. Cano, O. de Barbieri, and F. Engelmann, Nucl. Fusion 23, 1153 (1983). 